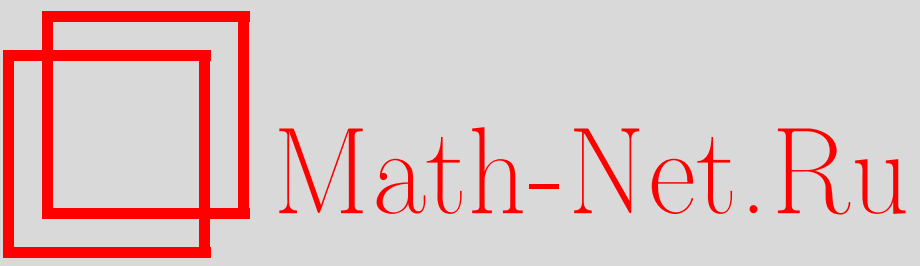

М. В. Козлов, О больших уклонениях строго докритических ветвящихся процессов в случайной среде с геометрическим распределением числа потомков, Теория вероятн. и ее примен., 2009, том 54, выпуск 3, 439-465

DOI: https://doi.org/10.4213/tvp2804

Использование Общероссийского математического портала Math-Net.Ru подразумевает, что вы прочитали и согласны с пользовательским соглашением

http://www.mathnet.ru/rus/agreement

Параметры загрузки:

IP : 54.174 .149 .18

26 апреля 2023 г., 02:52:47 
2009

(C) 2009 г.

КОЗЛОВ М. В.*

\title{
О БОЛЬШИХ УКЛОНЕНИЯХ СТРОГО ДОКРИТИЧЕСКИХ ВЕТВЯЩИХСЯ ПРОЦЕССОВ В СЛУЧАЙНОЙ СРЕДЕ С ГЕОМЕТРИЧЕСКИМ РАСПРЕДЕЛЕНИЕМ ЧИСЛА ПОТОМКОВ $^{1)}$
}

\begin{abstract}
Для строго докритического ветвящегося процесса $Z_{n}$ с геометрическим распределением числа непосредственных потомков в случайной среде $X_{n}$ из независимых одинаково распределенных величин найдена асимптотика вероятностей больших уклонений $\mathbf{P}\left(\ln Z_{n}>t n\right)$ при $0<t \leqslant \mu^{*}$ в предположении, что шаг $X_{i}$ сопровождающего случайного блуждания $S_{n}=\sum_{i=1}^{n} X_{i}$ удовлетворяет правостороннему условию Крамера. Она экспоненциальна по $n \mathrm{c}$ множителем при $n$, линейно зависящим от $t$. Ранее автором было показано, что при $t>\mu^{*}$ отношение вероятностей $\mathbf{P}\left(\ln Z_{n}>t n\right)$ и $\mathbf{P}\left(S_{n}>t n\right)$ стремится при $n \rightarrow \infty$ к положительной постоянной. Критическое значение $\mu^{*}$ параметра $t$ равняется производной преобразования Лапласа $\theta(\lambda)=\mathbf{E} e^{\lambda X_{1}}$ в точке $\lambda^{*}>1$, для которой $\theta\left(\lambda^{*}\right)=\theta(1)$. Для $t>\mu^{*}$ большие уклонения процесса $Z_{n}$ возникают за счет больших уклонений сопровождающего случайного блуждания. Для $t<\mu^{*}$ реализация больших уклонений протекает иначе: до случайного момента $T_{n}=n \widehat{s_{t}}+O_{p}(1) \sqrt{n}, \widehat{s}_{t}:=1-t / \mu^{*}$, от процесса $Z_{n}$ требуется лишь, чтобы он не вырождался, а на участке $\left[T_{n}, n\right]$ ему предписывается большое уклонение на величину порядка $\mu^{*} n\left(1-\widehat{s}_{t}\right)=t n$, которое реализуется так же, как и в случае $t>\mu^{*}$.

Ключевые слова и фразы: ветвящиеся процессы, случайная среда, большие уклонения, случайное блуждание, условие Крамера, экспоненциальный функционал.
\end{abstract}

1. Введение. Семейство производящих функций, отвечающих геометрическому распределению вероятностей, параметризуем следующим удобным для нас образом: $g_{x}(z):=1-\left(1+e^{-x}(1-z)^{-1}\right)^{-1}, 0 \leqslant z \leqslant 1$, где $x$ пробегает множество действительных чисел. Для любой последовательности $x_{n}, n=1,2, \ldots$, значений параметра определим ветвящийся процесс Гальтона-Ватсона в «изменяющейся среде» $\left(x_{n}\right)$ как неоднородную марковскую цепь на множестве целых неотрицательных чисел с

\footnotetext{
*Московский государственный университет им. М.В. Ломоносова, механикоматематический факультет, Ленинские горы, 119992 Москва, Россия; e-mail: kozlov_mikhail@mail.ru

1) Работа выполнена при поддержке РФФИ (проект № 07-01-00077).
} 
переходными вероятностями $p_{i j}\left(x_{n}\right)$ на $n$-м шаге, находящимися из соотношений: $\sum_{j=0}^{\infty} p_{i j}\left(x_{n}\right) z^{j}=\left(g_{x_{n}}(z)\right)^{i}, i=0,1,2, \ldots$ Введем «случайную среду» как последовательность $X_{n}, n=1,2, \ldots$, независимых одинаково распределенных случайных величин (н.о.р. сл.в.) и будем использовать $X$ в качестве общего обозначения для сл.в. $X_{n}$.

Пусть функция распределения $F(x)$ сл.в. $X$ невырождена, а математическое ожидание $\mathbf{E} X=: \mu_{0}$ конечно. Ветвящийся процесс $Z_{n}$ в случайной среде $X_{n}$ (ВПСС) определим как смесь процессов Гальтона-Ватсона в изменяющейся среде по мере, индуцируемой на последовательностях $\left(x_{n}\right)$ случайной средой. Положим $Z_{0}=1$. Процесс $Z_{n}$ является марковской цепью с переходными вероятностями $p_{i j}:=\mathbf{E} p_{i j}(X)$. Случайное блуждание $S_{n}:=\sum_{i=1}^{n} X_{i}, n=1,2, \ldots, S_{0}=0$, называют сопровождающим.

Положив $s_{j}:=x_{1}+\cdots+x_{j}, j \geqslant 1, s_{0}=0$, имеем [1]:

$$
g_{x_{1}}\left(\ldots\left(g_{x_{n}}(z) \ldots\right)\right)=1-\left((1-z)^{-1} e^{-s_{n}}+\sum_{j=0}^{n-1} e^{-s_{j}}\right)^{-1} \text {. }
$$

Учитывая, что хвосты распределения вероятностей с производящей функцией $g(z)$ имеют производящую функцию $(1-g(z)) /(1-z)$, находим для любого натурального $N$ :

$$
\begin{aligned}
& \mathbf{P}\left(Z_{n}>N\right)=\mathbf{E}\left\{\left(\sum_{j=0}^{n} e^{-S_{j}}\right)^{-1}\left(1+e^{-S_{n}}\left(\sum_{j=0}^{n-1} e^{-S_{j}}\right)^{-1}\right)^{-N}\right\} \\
& =\int \cdots \int\left(\sum_{j=0}^{n} e^{-s_{j}}\right)^{-1}\left(1+e^{-s_{n}}\left(\sum_{j=0}^{n-1} e^{-s_{j}}\right)^{-1}\right)^{-N} \prod_{i=1}^{n} d F\left(x_{i}\right) .
\end{aligned}
$$

Исследование вероятностей больших уклонений процесса $Z_{n}$ сводится к изучению асимптотики интегрального функционала (1.1) от траектории сопровождающего случайного блуждания при $n, N \rightarrow \infty$.

Предположим, что выполнено правостороннее условие Крамера:

$$
\mathbf{E} e^{\lambda X}=: \theta(\lambda)<\infty \quad \text { при } 0<\lambda \leqslant \lambda^{+}, \lambda^{+}>0,
$$

и $-\infty<\mu_{0}<\infty$. Тогда, как известно [2], в некотором интервале значений $t>\mu_{0}$ имеет место следующая асимптотика вероятностей больших уклонений:

$$
\mathbf{P}\left(S_{n}>t n\right) \sim c(t) n^{-1 / 2} e^{-\psi(t) n}, \quad n \rightarrow \infty,
$$

где $c(t)>0$, а $\psi(t)>0$ - так называемая функция отклонений (см. ниже пп. $2 \mathbf{a}, 2 \mathbf{c})$, символ $\sim$ означает, что отношение соединенных им выражений стремится к 1. Асимптотика (1.2) лежит в основе исследований вероятностей больших уклонений типа $\ln Z_{n}>t n$. В работе автора [3] показано, что вероятности больших уклонений $\mathbf{P}\left(\ln Z_{n}>t n\right)$ имеют с 
точностью до постоянного множителя такую же асимптотику (1.2), что и сопровождающее случайное блуждание, в случаях ВПСС надкритических: $\mu_{0}>0$, критических: $\mu_{0}=0$, слабо и промежуточно докритических: $\mu_{0}<0$ и, соответственно, $\theta^{\prime}(1)>0$ или $\theta^{\prime}(1)=0$. ВПСС называется строго докритическим, если $\theta^{\prime}(1)<0\left(\mu_{0}<0\right)$. В предположении

$$
\begin{gathered}
\theta^{\prime}(1)<0 \text { и существует } \lambda^{*} \in\left(1, \lambda^{+}\right) \text {такое } \\
\text { что } \theta\left(\lambda^{*}\right)=\theta(1), \mu_{0}>-\infty,
\end{gathered}
$$

асимптотика вероятностей $\mathbf{P}\left(\ln Z_{n}>t n\right)$ сохраняет вид (1.2) для $t>$ $\mu^{*}=:\left.(d / d \lambda) \theta(\lambda)\right|_{\lambda=\lambda^{*}}[3]$. Далее исследуются вероятности больших уклонений строго докритических ВПСС в промежутке $\left(0, \mu^{*}\right]$ изменения $t$. Ввиду ограничений на объем статьи исследование проводится в предположении

распределение сл.в. $X$ нерешетчатое.

Изменения, которые следует внести в случае решетчатого распределения, не существенны.

Предположим, что выполнены условия (A0), (A1), $-\infty<\mu_{0}<0$ и существует $\kappa>0$ такое, что $\theta(\kappa)=1, \mathbf{E}\left(|X| e^{\kappa X}\right)<\infty$. Введем сл.в. $U_{n}:=\sum_{j=0}^{n} e^{S_{j}}, n \leqslant \infty$. В [4] найдена асимптотика $\mathbf{P}\left(U_{\infty}>x\right) \sim C_{1} x^{-\kappa}$, $x \rightarrow \infty$, из которой следует, что $\mathbf{E} U_{\infty}^{\lambda}<\infty$ при $\lambda<\kappa$ и $\mathbf{E} U_{\infty}^{\kappa}=\infty$. В этой связи может представлять независимый интерес соотношение $\mathbf{E} U_{n}^{\kappa} \sim C_{3} n, n \rightarrow \infty\left(\mathbf{E} U_{n}^{\kappa}<\infty\right.$, поскольку $\left.\theta(\kappa)<\infty\right)$. Его доказательство (теорема 3 ) служит введением в более трудоемкое доказательство основного результата работы (теоремы 4,5 ). Отметим, что совсем просто выводится соотношение $\mathbf{E} e^{\kappa M_{n}} \sim C_{2} n, n \rightarrow \infty, M_{n}:=\max _{0 \leqslant j \leqslant n} S_{j}$ (теорема 2).

При ссылках на подпункты внутри пункта, занумерованного арабской цифрой, указывается только буква: $\mathbf{a}, \mathbf{b}, \mathbf{c}, \ldots$, а за его пределами также и цифра: $2 \mathbf{a}, 4 \mathbf{b}, \ldots$.

\section{2. Обозначения и предварительные сведения.}

а. Положим $\theta(\lambda):=\mathbf{E} e^{\lambda X}, \lambda \in\left[0, \lambda^{+}\right], \mu(\lambda) \equiv \mu_{\lambda}:=(d / d \lambda) \ln \theta(\lambda)=$ $\theta^{\prime}(\lambda) / \theta(\lambda)$. Так как $(d / d \lambda) \mu(\lambda)=: \sigma^{2}(\lambda)>0$, то функция $\mu(\lambda) \equiv \mu_{\lambda}$ строго возрастает и существует обратная к ней $\lambda(\mu) \equiv \lambda_{\mu}$, определенная на $\left[\mu_{0}, \mu^{+}\right), \mu^{+}:=\lim _{\lambda \rightarrow \lambda^{+}} \mu(\lambda) \leqslant \infty, \mu_{0}=\mathbf{E} X$. Предположим для определенности, что $\mu^{+}<\infty$. Случай $\mu^{+}=\infty$ не приводит к дополнительным осложнениям. Положим для краткости $\mu^{*}:=\mu\left(\lambda^{*}\right)$ (см. (A2)). Введем для сл.в. $X$ функиию отклонений

$$
\psi(t):=t \lambda_{t}-\ln \theta\left(\lambda_{t}\right)=\sup _{\lambda}\{t \lambda-\ln \theta(\lambda)\}, \quad t \in\left(\mu_{0}, \mu^{+}\right) .
$$


Легко видеть, что $(d / d t) \psi(t)=\lambda(t),\left(d^{2} / d t^{2}\right) \psi(t)=\left(\sigma^{2}\left(\lambda_{t}\right)\right)^{-1}$. Положим

$$
\psi(t ; \lambda):=\psi\left(\mu_{\lambda}\right)+\lambda\left(t-\mu_{\lambda}\right) \equiv \lambda t-\ln \theta(\lambda), \quad \widehat{\psi}(t):=\psi\left(t ; \lambda^{*}\right) .
$$

Функция $\psi(t ; \lambda)$ представляет касательную к графику $\psi(t)$ в точке $\mu_{\lambda}$.

b. Введем вспомогательную переменную $\nu \in\left(\mu_{0}, \mu^{+}\right)$и при фиксированном $\nu$ - последовательность $X^{(\nu)}, X_{1}^{(\nu)}, X_{2}^{(\nu)}, \ldots$ н.о.p. сл.в. с функцией распределения $F^{(\nu)}(x):=\theta\left(\lambda_{\nu}\right)^{-1} \int_{-\infty}^{x} e^{\lambda_{\nu} y} d F(y)$. Заметим, что

$$
\begin{array}{ll}
\theta^{(\nu)}(\lambda):=\mathbf{E} e^{\lambda X^{(\nu)}}=\frac{\theta\left(\lambda_{\nu}+\lambda\right)}{\theta\left(\lambda_{\nu}\right)}, & \lambda \in\left[-\lambda_{\nu}, \lambda^{+}-\lambda_{\nu}\right], \\
\mu^{(\nu)}(\lambda):=\frac{d}{d \lambda} \theta^{(\nu)}(\lambda)=\mu\left(\lambda_{\nu}+\lambda\right), & \mathbf{E} X^{(\nu)}=\nu, \quad \mathbf{D} X^{(\nu)}=\sigma^{2}\left(\lambda_{\nu}\right) .
\end{array}
$$

Функция $\lambda^{(\nu)}(\mu) \equiv \lambda_{\mu}^{(\nu)}$, обратная к $\mu^{(\nu)}(\lambda)$, равна $\lambda(\mu)-\lambda(\nu)$, функция отклонений для сл.в. $X^{(\nu)}$ равна

$$
\psi^{(\nu)}(t)=t \lambda_{t}^{(\nu)}-\ln \theta^{(\nu)}\left(\lambda_{t}^{(\nu)}\right)=\psi(t)-\psi(\nu)-(t-\nu) \lambda_{\nu}, \quad t \in\left(\mu_{0}, \mu^{+}\right) .
$$

Положим

$$
\begin{aligned}
& \bar{X}^{(\nu)}:=-X^{(\nu)}, \quad \bar{\theta}^{(\nu)}(\lambda)=\theta^{(\nu)}(-\lambda), \quad \bar{\mu}^{(\nu)}(\lambda)=-\mu^{(\nu)}(-\lambda), \\
& \bar{\lambda}^{(\nu)}(\mu)=-\lambda^{(\nu)}(-\mu), \quad \bar{\psi}^{(\nu)}(t)=\psi^{(\nu)}(-t), \\
& \lambda \in\left(-\lambda^{+}+\lambda_{\nu}, \lambda_{\nu}\right], \quad \mu \in\left(-\mu^{+},-\mu_{0}\right), \quad t \in\left(-\mu^{+},-\mu_{0}\right) .
\end{aligned}
$$

c. Положим

$$
S_{n}^{(\nu)}:=\sum_{i=1}^{n} X_{i}^{(\nu)}, \quad \bar{S}_{n}^{(\nu)}:=\sum_{i=1}^{n} \bar{X}_{i}^{(\nu)}, \quad S_{0}^{(\nu)}=\bar{S}_{0}^{(\nu)}=0
$$

и выпишем оценки для хвостов распределений вероятностей:

$$
\begin{aligned}
& \mathbf{P}\left(S_{n}>x\right)=\mathbf{P}\left(e^{\lambda S_{n}}>e^{\lambda x}\right) \leqslant e^{-\lambda x} \theta(\lambda)^{n} \text { при } x>0, \lambda \in\left(0, \lambda^{+}\right), \\
& \mathbf{P}\left(S_{n}>t n\right)=\mathbf{P}\left(e^{\lambda\left(t_{1}\right) S_{n}}>e^{\lambda\left(t_{1}\right) t n}\right) \leqslant e^{-\psi\left(t ; \lambda\left(t_{1}\right)\right) n} \text { при } t \geqslant t_{1} \in\left(\mu_{0}, \mu^{+}\right), \\
& \mathbf{P}\left(S_{n}>t n\right) \leqslant e^{-\psi(t) n}, \quad t \in\left(\mu_{0}, \mu^{+}\right), \\
& \mathbf{P}\left(S_{n}^{(\nu)}>t n\right) \leqslant e^{-\psi^{(\nu)}(t) n}, \quad t \in\left(\nu, \mu^{+}\right), \\
& \mathbf{P}\left(S_{n}^{(\nu)}>t n\right) \leqslant e^{-\psi^{(\nu)}\left(t ; \lambda^{(\nu)}\left(t_{1}\right)\right) n}, \quad t \geqslant t_{1} \in\left(\nu, \mu^{+}\right), \\
& \mathbf{P}\left(S_{n}^{(\nu)}<t n\right) \leqslant e^{-\psi^{(\nu)}(t) n}, \quad t \in\left(\mu_{0}, \nu\right), \\
& \mathbf{P}\left(S_{n}^{(\nu)}<t n\right) \leqslant e^{-\psi^{(\nu)}\left(t ; \lambda^{(\nu)}\left(t_{1}\right)\right) n}, \quad t \leqslant t_{1} \in\left(\mu_{0}, \nu\right),
\end{aligned}
$$

где $\psi^{(\nu)}(t ; \lambda):=\lambda t-\ln \theta^{(\nu)}(\lambda)$.

Соотношение (1.2) выполняется равномерно по $t$ из любого отрезка, содержащегося в $\left(\mu_{0}, \mu^{+}\right)$, а $c(t)=\left(\sqrt{2 \pi} \lambda_{t} \sigma\left(\lambda_{t}\right)\right)^{-1}$ в предположении (A0). 
d. Для хвостов распределений максимума и минимума случайных блужданий имеют место следующие оценки (см. $[5, \S 22]$ или $[3$, лемма 1$])$ :

$$
\begin{aligned}
& \mathbf{P}\left(\max _{j \geqslant 0} S_{j} \geqslant y\right) \leqslant e^{-q y}, \quad q:=\sup \{\lambda: \theta(\lambda) \leqslant 1\}, \quad \mu_{0}<0 ; \\
& \mathbf{P}\left(\max _{j \geqslant 0} S_{j}^{(\nu)} \geqslant y\right) \leqslant e^{-q^{(\nu)} y}, \quad q^{(\nu)}:=\sup \left\{\lambda: \theta^{(\nu)}(\lambda) \leqslant 1\right\}, \quad \nu \in\left(\mu_{0}, 0\right) ; \\
& \mathbf{P}\left(\min _{j \geqslant 1} S_{j}^{(\nu)} \leqslant-y\right) \leqslant e^{\bar{q}^{(\nu)} y}, \quad \bar{q}^{(\nu)}:=\inf \left\{\lambda: \theta^{(\nu)}(\lambda) \leqslant 1\right\}, \quad \nu \in\left(0, \mu^{+}\right) .
\end{aligned}
$$

е. Функционал $\mathbf{E} h_{n}\left(S_{1}, \ldots, S_{n}\right)=\int \cdots \int h_{n}\left(s_{1}, \ldots, s_{n}\right) \prod_{i=1}^{n} d F\left(x_{i}\right)$ для измеримой функции $h_{n}$ от случайного блуждания после замены меры $\prod_{i=1}^{n} d F\left(x_{i}\right)=\theta\left(\lambda_{\nu}\right)^{n} e^{-\lambda_{\nu} s_{n}} \prod_{i=1}^{n} d F^{(\nu)}\left(x_{i}\right)$ преобразуется к виду

$$
\begin{array}{r}
\theta\left(\lambda_{\nu}\right)^{n} \int \cdots \int e^{-\lambda_{\nu} s_{n}} h_{n}\left(s_{1}, \ldots, s_{n}\right) \prod_{i=1}^{n} d F^{(\nu)}\left(x_{i}\right) \\
=e^{-\psi(\nu) n} \mathbf{E}\left\{e^{-\lambda_{\nu}\left(S_{n}^{(\nu)}-\nu n\right)} h_{n}\left(S_{1}^{(\nu)}, \ldots, S_{n}^{(\nu)}\right)\right\} .
\end{array}
$$

f. Для нерешетчатого распределения $F$ выпишем асимптотическое разложение функции распределения $F_{n}^{(\nu)}(\cdot)$ нормированной суммы $\left(S_{n}^{(\nu)}-\right.$ $\nu n) /\left(\sigma\left(\lambda_{\nu}\right) \sqrt{n}\right)[6]:$

$$
F_{n}^{(\nu)}(x)=\Phi(x)+\frac{a_{3}^{(\nu)}}{6 \sigma\left(\lambda_{\nu}\right)^{3} \sqrt{n}}\left(1-x^{2}\right) \varphi(x)+\frac{\varepsilon_{n}^{(\nu)}(x)}{\sqrt{n}},
$$

где $\Phi(x)$ и $\varphi(x)$ - функция распределения и плотность распределения стандартного нормального закона, $a_{3}^{(\nu)}=\mathbf{E}\left(X^{(\nu)}-\nu\right)^{3}, \sup _{x}\left|\varepsilon_{n}^{(\nu)}(x)\right|=$ $\varepsilon_{n}^{(\nu)} \rightarrow 0$ при $n \rightarrow \infty$. В работе [2] указано, что $\sup _{\alpha \leqslant \nu \leqslant \beta} \varepsilon_{n}^{(\nu)}=\varepsilon_{n} \rightarrow$ $0, n \rightarrow \infty$, при любых $\mu_{0}<\alpha<\beta<\mu^{+}$. Это утверждение легко проверить, дополнив вывод, приведенный в [6, гл. XVI, $\S \S 2-4]$, либо воспользовавшись доказательством теоремы 3.3 .1 из [7].

g. Воспользовавшись асимптотическим разложением из предыдущего пункта, получаем для вероятности $\mathbf{P}\left(S_{n}^{(\nu)}-\nu n \in[x, x+\Delta]\right), \Delta>0$, выражение

$$
\left.\Phi\left(\frac{y}{\sigma\left(\lambda_{\nu}\right) \sqrt{n}}\right)\right|_{x} ^{x+\Delta}+\left.\frac{a_{3}^{(\nu)}}{6 \sigma\left(\lambda_{\nu}\right)^{3} \sqrt{n}}\left(\left(1-\frac{y^{2}}{\sigma\left(\lambda_{\nu}\right)^{2} n}\right) \varphi\left(\frac{y}{\sigma\left(\lambda_{\nu}\right) \sqrt{n}}\right)\right)\right|_{x} ^{x+\Delta}
$$

плюс член $o\left(n^{-1 / 2}\right)$, равномерно малый по $\nu \in[\alpha, \beta] \subseteq\left(\mu_{0}, \mu^{+}\right)$и по $x$. Поскольку производная функции $\left(1-x^{2}\right) \varphi(x)$ ограничена, то второе слагаемое в указанном выражении есть $O\left(n^{-1}\right)$ равномерно по $\nu$ и по $x$, а первое представимо в виде:

$$
\frac{\Delta}{\sqrt{2 \pi} \sigma\left(\lambda_{\nu}\right) \sqrt{n}} \exp \left\{-\frac{x^{2}}{2 \sigma\left(\lambda_{\nu}\right)^{2} n}-\frac{x \cdot \theta \Delta}{\sigma\left(\lambda_{\nu}\right)^{2} n}-\frac{(\theta \Delta)^{2}}{2 \sigma\left(\lambda_{\nu}\right)^{2} n}\right\}
$$


где $\theta \equiv \theta(x, \Delta, \nu) \in[0,1]$. В итоге для рассматриваемой вероятности получаем асимптотическую формулу

$$
\Delta\left(\sigma\left(\lambda_{\nu}\right) \sqrt{2 \pi n}\right)^{-1} \exp \left\{-\frac{x^{2}}{2 \sigma\left(\lambda_{\nu}\right)^{2} n}\right\}+\delta_{n}^{(\nu)}(x) n^{-1 / 2},
$$

где $\delta_{n}^{(\nu)}(x) \rightarrow 0$ при $n \rightarrow \infty$ равномерно по $\nu \in[\alpha, \beta] \subseteq\left(\mu_{0}, \mu^{+}\right)$и по $x=o(n)$ (т.е. $\left.|x| \leqslant b_{n}=o(n)\right)$. Аналогично получаем, что для $\nu \in[\alpha, \beta]$ и всех $x$

$$
\mathbf{P}\left(S_{n}^{(\nu)}-\nu n \in[x, x+\Delta]\right) \leqslant\left(\Delta+\delta_{n}\right)\left(\sigma\left(\lambda_{\nu}\right) \sqrt{2 \pi n}\right)^{-1},
$$

где последовательность $\delta_{n} \rightarrow 0$ при $n \rightarrow \infty$ мы выберем убывающей.

3. Основные результаты. Чтобы облегчить знакомство с работой, вывод точной асимптотики вероятностей больших уклонений $\mathbf{P}\left(\ln Z_{n}>t n\right)$ разбивается на несколько этапов, из которых первый получение логарифмической асимптотики этих вероятностей.

Теорема 1. В предположениях (A0)-(A2) для любого $0<\varepsilon<\mu^{*} u$ $t \in\left[\varepsilon, \mu^{*}\right]$ выполнены неравенства

$$
c_{\varepsilon} n^{-1} \leqslant e^{\hat{\psi}(t) n} \mathbf{P}\left(\ln Z_{n}>t n\right) \leqslant C_{\varepsilon} n^{\lambda_{t}}
$$

для некоторых $0<c_{\varepsilon}<C_{\varepsilon}<\infty$ (задаваемьх конструктивно, причем $c_{\varepsilon}, C_{\varepsilon} \rightarrow 0$ при $\left.\varepsilon \rightarrow 0\right)$, где $\widehat{\psi}(t)=\lambda^{*} t-\ln \theta\left(\lambda^{*}\right)($ (cм. n. 2a).

Теорема 2. Пусть выполнено (A1), $-\infty<\mu_{0}<0$ и существует $\kappa>0$ mакое, ито $\theta(\kappa)=1$. Тогда при $n \rightarrow \infty \mathbf{E} e^{\kappa M_{n}} \sim C_{2} n, M_{n}=$ $\max _{0 \leqslant j \leqslant n} S_{j}$, где постоянная $C_{2}>0$ определена в конче $n .5$.

Теорема 3. В условиях теоремь 2 при $n \rightarrow \infty$ имеет место соотношение; $\mathbf{E} U_{n}^{\kappa} \sim C_{3} n, U_{n}=\sum_{j=0}^{n} e^{S_{j}}$, выражение для $C_{3}$ приводится в (6.10). Тогда

Теорема 4. Пусть выполнень условия (А0)-(A2) $u-\infty<\mu_{0}<0$.

$$
\mathbf{P}\left(\ln Z_{n}>\mu^{*} n\right) \sim\left(2 \mu^{*}\right)^{-1} H e^{-\psi\left(\mu^{*}\right) n}, \quad n \rightarrow \infty .
$$

Теорема 5. В предположениях теоремы 4

$$
\mathbf{P}\left(\ln Z_{n}>t n\right) \sim \Phi\left(\sqrt{n} \frac{\mu^{*}-t}{\sigma\left(\lambda^{*}\right)} \sqrt{\frac{\mu^{*}}{t}}\right)\left(\mu^{*}\right)^{-1} H e^{-\hat{\psi}(t) n}, \quad n \rightarrow \infty,
$$

равномерно по $t \in\left[\varepsilon, \mu^{*}\right]$, где $0<\varepsilon<\mu^{*}-$ произвольное.

Выражение для постоянной $H$ приведено в (7.30). Первый множитель в правой части (3.3) лежит в промежутке от $1 / 2$ до 1 и может быть заменен на 1 при $t=t_{n}$ таких, что $\left(\mu^{*}-t_{n}\right) \sqrt{n} \rightarrow \infty, n \rightarrow \infty$, и на $1 / 2$ при $\mu^{*}-t_{n}=o(1 / \sqrt{n})$. Таким образом, теорема 4 является частным случаем теоремы 5 . В п. 7 доказывается теорема 4 и делаются необходимые замечания относительно доказательства теоремы 5. 


\section{4. Доказательство теоремы 1.}

a. Введем обозначения $l_{n}:=\min _{0 \leqslant i \leqslant n} s_{i}, k_{n}:=\min \left(k \leqslant n: s_{k}=l_{n}\right)$, $L_{n}:=\min _{0 \leqslant i \leqslant n} S_{i}, K_{n}:=\min \left(k \leqslant n: S_{k}=L_{n}\right)$. Оценивая суммы $\sum_{j=0}^{n} e^{-s_{j}}, \sum_{j=0}^{n-1} e^{-s_{j}}$ снизу максимальным слагаемым, а сверху - его произведением на число слагаемых в сумме, получим для подынтегральной функции в (1.1) с учетом неравенства $\sum_{j=0}^{n-1} e^{-s_{j}} \leqslant \sum_{j=0}^{n} e^{-s_{j}}$ оценки соответственно сверху и снизу:

$$
e^{l_{n}}\left(1+n^{-1} e^{l_{n}-s_{n}}\right)^{-N} \quad \text { и } \quad(n+1)^{-1} e^{l_{n}}\left(1+e^{l_{n-1}-s_{n}}\right)^{-N} .
$$

Для $t \in\left(0, \mu^{+}\right)$положим $N_{t}:=\left[e^{t n}\right]$, где [·] обозначает целую часть заключенного в скобки числа. В основе доказательства теоремы 1 лежит исследование интеграла (1.1), в котором подынтегральная функция заменяется на $e^{l_{n}}\left(1+e^{l_{n}-s_{n}}\right)^{-N_{t}}$. Этот интеграл запишем в виде следующей суммы математических ожиданий:

$$
\sum_{m=0}^{n} \mathbf{E}\left(e^{L_{n}}\left(1+e^{L_{n}-S_{n}}\right)^{-N_{t}} ; K_{n}=m\right)
$$

Ее слагаемые представим в виде произведения:

$$
\mathbf{E}\left(e^{L_{m}} ; K_{m}=m\right) \cdot \mathbf{E}\left(\left(1+e^{-S_{n-m}}\right)^{-N_{t}} ; L_{n-m}=0\right) .
$$

b. Покажем, что разность между математическими ожиданиями

$$
\mathbf{E}\left(e^{L_{n}}\left(1+e^{L_{n-1}-S_{n}}\right)^{-N_{t}}\right) \quad \text { и } \quad \mathbf{E}\left(e^{L_{n}}\left(1+e^{L_{n}-S_{n}}\right)^{-N_{t}}\right)
$$

убывает при $t \in[\alpha, \beta] \subseteq\left(0, \mu^{+}\right)$быстрее, чем $\exp \left(-e^{\gamma n}\right)$ при некотором $\gamma>0$; в таких случаях будем далее говорить, что скорость убывания двойная экспоненциальная по $n$. Первое из выражений (4.4) разложим в сумму математических ожиданий, суженных на события $\left\{L_{n-1}=L_{n}\right\}$ и $\left\{L_{n-1}>L_{n}\right\}$. Во втором случае имеем $L_{n}=S_{n}, L_{n-1}>S_{n}, e^{L_{n-1}-S_{n}}>1$, а соответствующее математическое ожидание не превосходит тем самым $2^{-N_{t}}$. В первом случае представим математическое ожидание в виде разности

$$
\mathbf{E}\left(e^{L_{n}}\left(1+e^{L_{n}-S_{n}}\right)^{-N_{t}}\right)-\mathbf{E}\left(e^{L_{n}}\left(1+e^{L_{n}-S_{n}}\right)^{-N_{t}} ; L_{n-1}>L_{n}\right) .
$$

Так как $\left\{L_{n-1}>L_{n}\right\} \subseteq\left\{L_{n}=S_{n}\right\}, L_{n}<0$, то вычитаемое не превосходит $2^{-N_{t}}$, и утверждение установлено.

c. Проверим, что при $\theta^{\prime}(1)<0\left(\mu_{0}<0\right)$

$$
\mathbf{E}\left(e^{L_{m}} ; K_{m}=m\right) \sim c_{1} \theta(1)^{m}, \quad m \rightarrow \infty, \quad c_{1}>0 .
$$


Запишем математическое ожидание (4.5) в интегральной форме:

$$
\begin{aligned}
& \int_{\left\{k_{m}=m\right\}} \ldots e e^{s_{m}} \prod_{i=1}^{m} d F\left(x_{i}\right)=\theta(1)^{m} \int_{\left\{s_{j}>s_{m},\right.} \ldots \int_{j<m\}} \prod_{i=1}^{m} d F^{\left(\mu_{1}\right)}\left(x_{i}\right) \\
& \quad=\theta(1)^{m} \mathbf{P}\left(S_{j}^{\left(\mu_{1}\right)}>S_{m}^{\left(\mu_{1}\right)}, j<m\right)=\theta(1)^{m} \mathbf{P}\left(S_{j}^{\left(\mu_{1}\right)}<0,1 \leqslant j \leqslant m\right) .
\end{aligned}
$$

Так как $\mu_{1}<0$, имеем $\mathbf{P}\left(S_{j}^{\left(\mu_{1}\right)}<0,1 \leqslant j \leqslant m\right) \rightarrow \mathbf{P}\left(S_{j}^{\left(\mu_{1}\right)}<0, j \geqslant 1\right)=$ : $c_{1}>0, m \rightarrow \infty$, и приходим к соотношению (4.5).

d. При $\mu_{0}<0$ для любого $\nu \in\left(0, \mu^{+}\right)$положим $L_{n}^{(\nu)}:=\min _{0 \leqslant i \leqslant n} S_{i}^{(\nu)}$, $L_{\infty}^{(\nu)}:=\min _{n \geqslant 0} S_{n}^{(\nu)}$. Проверим, что $\mathbf{P}\left(L_{n}^{(\nu)}=0\right) \rightarrow \mathbf{P}\left(L_{\infty}^{(\nu)}=0\right), n \rightarrow \infty$, равномерно по $\nu$ из любого отрезка, содержащегося в $\left(0, \mu^{+}\right)$. Разность $\mathbf{P}\left(L_{n}^{(\nu)}=0\right)-\mathbf{P}\left(L_{\infty}^{(\nu)}=0\right)=\mathbf{P}\left(L_{n}^{(\nu)}=0, \bigcup_{k=n+1}^{\infty}\left\{S_{k}^{(\nu)}<0\right\}\right)$ оценим сверху суммой $\sum_{k=n+1}^{\infty} \mathbf{P}\left(S_{k}^{(\nu)}<0\right)$. Применяя неравенство $\mathbf{P}\left(S_{k}^{(\nu)}<0\right) \leqslant$ $e^{-\psi^{(\nu)}(0) k}$ (см. п. 2с ) и используя непрерывность $\psi^{(\nu)}(t)$ по $\nu$ (см. п. 2b), приходим к требуемому заключению.

е. Покажем, что при $\mu_{0}<0$ для любого $\Delta>0$

$$
\mathbf{P}\left(S_{n}^{(\nu)}-\nu n \in[x, x+\Delta], L_{n}^{(\nu)}=0\right) \sim \Delta c_{2}^{(\nu)} n^{-1 / 2}, \quad n \rightarrow \infty,
$$

равномерно по $x=o(\sqrt{n})$ (т.е. $|x| \leqslant b_{n}=o(\sqrt{n})$ и по $\nu$ из любого отрезка $[\alpha, \beta]$, содержащегося в $\left(0, \mu^{+}\right) ; c_{2}^{(\nu)}:=\left(\sigma\left(\lambda_{\nu}\right) \sqrt{2 \pi}\right)^{-1} \mathbf{P}\left(L_{\infty}^{(\nu)}=0\right)$.

Положим $m_{n}=[\sqrt[4]{n}]$ и представим вероятность (4.6) в виде

$$
\mathbf{P}\left(S_{n}^{(\nu)}-\nu n \in[x, x+\Delta],\left\{L_{m_{n}}^{(\nu)}=0\right\} \backslash\left\{\bigcup_{j=m_{n}+1}^{n}\left\{S_{j}^{(\nu)}<0\right\}\right\}\right) .
$$

Оценим ее снизу разностью вероятностей

$$
\mathbf{P}\left(S_{n}^{(\nu)}-\nu n \in[x, x+\Delta], L_{m_{n}}^{(\nu)}=0\right)-\mathbf{P}\left(\bigcup_{j=m_{n}+1}^{n}\left\{S_{j}^{(\nu)}<0\right\}\right)
$$

а сверху - первым членом в (4.7). Второй член в (4.7) не превосходит

$$
\mathbf{P}\left(S_{m_{n}}^{(\nu)} \geqslant \frac{\nu m_{n}}{2}\right) \cdot \mathbf{P}\left(\min _{1 \leqslant j \leqslant n-m_{n}} S_{j}^{(\nu)}<\frac{\nu m_{n}}{2}\right)+\mathbf{P}\left(S_{m_{n}}^{(\nu)}<\frac{\nu m_{n}}{2}\right) .
$$

Второе слагаемое в (4.8) не превосходит величины $\exp \left(-\psi^{(\nu)}(\nu / 2) m_{n}\right)=$ $o\left(n^{-1 / 2}\right)$ равномерно по указанным выше $\nu$ (см. пп. $\left.2 \mathbf{c}, 2 \mathbf{b}\right)$. Второй сомножитель первого слагаемого в (4.8) не превосходит $\exp \left(\bar{q}^{(\nu)} \nu m_{n} / 2\right)$, что также есть $o\left(n^{-1 / 2}\right)$ равномерно по $\nu$ (см. п. $2 \mathbf{d}$ ).

Первую вероятность в (4.7) запишем в интегральной форме:

$$
\int_{-\infty}^{\infty} \mathbf{P}\left(S_{n-m_{n}}^{(\nu)}-\nu\left(n-m_{n}\right)+y \in[x, x+\Delta]\right) \mathbf{P}\left(S_{m_{n}}^{(\nu)}-\nu m_{n} \in d y ; L_{m_{n}}^{(\nu)}=0\right)
$$


и разобьем область интегрирования в (4.9) на части $|y| \leqslant \delta m_{n}$ и $|y|>$ $\delta m_{n}, \delta>0$. В интеграле (4.9) по области $|y| \leqslant \delta m_{n}$ заменим подынтегральную функцию на равномерно по $\nu \in[\alpha, \beta] \subseteq\left(0, \mu^{+}\right)$эквивалентное выражение $\Delta\left(\sigma\left(\lambda_{\nu}\right) \sqrt{2 \pi n}\right)^{-1}$ (см. п. $\left.2 \mathbf{g}\right)$ и вынесем его за знак интеграла. Остающийся интеграл равен $\mathbf{P}\left(L_{m_{n}}^{(\nu)}=0\right)+o(1)$ и при $n \rightarrow \infty$ стремится к $\mathbf{P}\left(L_{\infty}^{(\nu)}=0\right)$ также равномерно по $\nu$. Интеграл (4.9) по области $|y|>\delta m_{n}$ оценим сверху вероятностью

$$
\mathbf{P}\left(\left|S_{m_{n}}^{(\nu)}-\nu m_{n}\right|>\delta m_{n}\right) \leqslant \exp \left\{-\left(\psi^{(\nu)}(\nu+\delta)+\psi^{(\nu)}(\nu-\delta)\right) m_{n}\right\},
$$

предполагая, что $\nu \pm \delta \in\left(\mu_{0}, \mu^{+}\right)$(см. п. 2c). Минимизируя выражения (см. п. 2b) $\psi^{(\nu)}(\nu+\delta)=\psi(\nu+\delta)-\psi(\nu)-\delta \lambda_{\nu}$ и $\psi^{(\nu)}(\nu-\delta)=\psi(\nu-\delta)-$ $\psi(\nu)+\delta \lambda_{\nu}$ по $\nu \in[\alpha, \beta] \subseteq\left(0, \mu^{+}\right)$при $\delta<\min \left(\alpha, \mu^{+}-\beta\right)$, получаем для рассматриваемого интеграла равномерную по $\nu$ оценку $o\left(n^{-1 / 2}\right)$. Таким образом, соотношение (4.6) установлено.

f. Пусть $\mu_{0}<0$, функция $h(x) \geqslant 0$ непосредственно интегрируема по Риману, $c_{2}^{(\nu)}:=\left(\sigma\left(\lambda_{\nu}\right) \sqrt{2 \pi}\right)^{-1} \mathbf{P}\left(L_{\infty}^{(\nu)}=0\right)$. Покажем, что

$$
\int_{-\infty}^{\infty} h(x) d \mathbf{P}\left(S_{n}^{(\nu)}-\nu n \leqslant x, L_{n}^{(\nu)}=0\right) \sim \frac{c_{2}^{(\nu)}}{\sqrt{n}} \int_{-\infty}^{\infty} h(x) d x, \quad n \rightarrow \infty,
$$

равномерно по $\nu \in[\alpha, \beta] \subseteq\left(0, \mu^{+}\right)$. Докажем вариант соотношения (4.10), сократив область интегрирования до $[0, \infty]$. Возьмем $B>0$ сколь угодно большим и разобьем соответствующий интеграл в сумму двух: $J_{1}^{(\nu)}$ по отрезку $[0, B]$ и $J_{2}^{(\nu)}$ по интервалу $(B, \infty)$. Возьмем разбиение $x_{0}=0<x_{1}<x_{2}<\cdots$ положительной полупрямой с шагом $\Delta$ и будем считать, что $B$ кратно $\Delta$. Обозначим через $\bar{m}_{k}$ и $\underline{m}_{k}$ супремум и инфимум функции $h(x)$ по отрезку $\left[x_{k-1}, x_{k}\right]$. Применяя (4.6), получим для верхней суммы Дарбу-Стилтьеса по отрезку $[0, B]$ равномерную по $\nu \in[\alpha, \beta]$ оценку:

$$
\sum_{x_{k} \leqslant B} \bar{m}_{k} \mathbf{P}\left(S_{n}^{(\nu)}-\nu n \in\left[x_{k-1}, x_{k}\right], L_{n}^{(\nu)}=0\right) \sim \frac{c_{2}^{(\nu)}}{\sqrt{n}} \sum_{x_{k} \leqslant B} \bar{m}_{k} \Delta, \quad n \rightarrow \infty .
$$

Аналогичную оценку получим для нижней суммы. Выбирая $\Delta>0$ достаточно малым, получим, что разность $\sum_{x_{k} \leqslant B}\left(\bar{m}_{k}-\underline{m}_{k}\right) \Delta$ между нижней и верхней суммами будет сколь угодно мала. Отсюда следует, что для всех достаточно больших $n$ и $\nu \in[\alpha, \beta]$ интеграл $J_{1}^{(\nu)}$ лежит в границах $\left(c_{2}^{(\nu)} \pm \varepsilon\right) n^{-1 / 2} \int_{0}^{B} h(x) d x$. Для оценивания интеграла $J_{2}^{(\nu)}$ также переходим к суммам Дарбу-Стилтьеса и используем неравенства (см. п. 2g)

$$
\mathbf{P}\left(S_{n}^{(\nu)}-\nu n \in\left[x_{k}, x_{k+1}\right]\right) \leqslant\left(\Delta+\delta_{n}\right)\left(\sigma\left(\lambda_{\nu}\right) \sqrt{2 \pi n}\right)^{-1} .
$$

В результате для достаточно малых $\Delta>0$, достаточно большого $B$ и всех достаточно больших $n$ модуль интеграла $J_{2}^{(\nu)}$ оценивается сверху 
равномерно по $\nu$ величиной $\varepsilon / \sqrt{n}$ для любого заранее заданного $\varepsilon>0$. Повторяя проведенные рассуждения для интеграла, взятого по области $(-\infty, 0)$, получаем $(4.10)$.

g. Покажем, что при $\theta^{\prime}(1)<0$ равномерно по $\nu \in[\alpha, \beta] \subseteq\left(0, \mu^{+}\right)$

$$
\mathbf{E}\left(\left(1+e^{-S_{n}}\right)^{-N_{\nu}} ; L_{n}=0\right) \sim c_{3}^{(\nu)} n^{-1 / 2} e^{-\psi(\nu) n}, \quad n \rightarrow \infty,
$$

где $c_{3}^{(\nu)}:=\left(\sigma\left(\lambda_{\nu}\right) \sqrt{2 \pi}\right)^{-1} \mathbf{P}\left(L_{\infty}^{(\nu)}=0\right) \Gamma\left(\lambda_{\nu}\right)$ и $\Gamma(\cdot)$ есть гамма-функция.

Математическое ожидание в (4.11) запишем в интегральной форме; сделав замену меры (см. п. 2е), получаем для него выражение

$$
\begin{array}{rl}
e^{-\psi(\nu) n} & \mathbf{E}\left\{e^{-\lambda_{\nu}\left(S_{n}^{(\nu)}-\nu n\right)}\left(1+e^{-S_{n}^{(\nu)}}\right)^{-N_{\nu}} ; L_{n}^{(\nu)}=0\right\} \\
\quad= & e^{-\psi(\nu) n} \int_{-\infty}^{\infty} e^{-\lambda_{\nu}(x-\nu n)}\left(1+e^{-x}\right)^{-N_{\nu}} \mathbf{P}\left(S_{n}^{(\nu)} \in d x ; L_{n}^{(\nu)}=0\right) .
\end{array}
$$

В интеграле (4.12) перейдем к переменной $y=x-\nu n$. Заметим, что

$$
\left(1+e^{-\nu n} e^{-y}\right)^{-N_{\nu}} \sim \exp \left(-e^{-y}\right), \quad n \rightarrow \infty,
$$

равномерно по $\nu \geqslant \alpha>0, y \geqslant-\delta n, 0<\delta<\alpha / 2$, а при $y \leqslant-\delta n$ выражение в левой части (4.13) убывает как двойная экспонента от $n$. Следовательно, для получения асимптотики (4.11) достаточно рассмотреть интеграл в (4.12) по области $x-\nu n \geqslant-\delta n$. Воспользовавшись эквивалентностью (4.13), приходим к интегралу

$$
\int_{-\delta n}^{\infty} \exp \left(-\lambda_{\nu} y-e^{-y}\right) \mathbf{P}\left(S_{n}^{(\nu)}-\nu n \in d y ; L_{n}^{(\nu)}=0\right) .
$$

Подынтегральная функция в (4.14) при $y \leqslant-\delta n$ оценивается для достаточно больших $n$ сверху величиной $\exp \left(-e^{\gamma n}\right), \gamma>0$. Поэтому расширение области интегрирования в (4.14) до всей прямой не повлияет на асимптотику. Применяя соотношение (4.10) к интегралу (4.14), взятому по всей прямой, приходим к результату (4.11). При этом $c_{3}^{(\nu)}=c_{2}^{(\nu)} \int_{-\infty}^{\infty} \exp \left(-\lambda_{\nu} y-e^{-y}\right) d y$. Замена переменной $x=e^{-y}$ показывает, что последний интеграл есть гамма-функция $\Gamma\left(\lambda_{\nu}\right)$.

h. При $\theta^{\prime}(1)<0$ равномерно по $\nu \in[\alpha, \beta] \subseteq\left(0, \mu^{+}\right)$

$$
\mathbf{E}\left(\left(1+n^{-1} e^{-S_{n}}\right)^{-N_{\nu}} ; L_{n}=0\right) \sim c_{3}^{(\nu)} n^{\lambda_{\nu}-1 / 2} e^{-\psi(\nu) n}, \quad n \rightarrow \infty .
$$

Вывод аналогичен (4.11), только вместо интеграла (4.14) получаем интеграл

$$
\int_{-\delta n}^{\infty} \exp \left(-\lambda_{\nu} y-n^{-1} e^{-y}\right) \mathbf{P}\left(S_{n}^{(\nu)}-\nu n \in d y ; L_{n}^{(\nu)}=0\right) .
$$

Далее делаем замену переменной $y=x-\ln n$, в результате чего к асимптотике (4.11) добавляется множитель $e^{\lambda_{\nu} \ln n}=n^{\lambda_{\nu}}$. 
i. Используем асимптотики (4.5) и (4.11) для сомножителей (4.3) при $m \rightarrow \infty, m \leqslant\left(1-t / \mu^{+}-\varepsilon\right) n$ и $\varepsilon>0$ сколь угодно малом. Границы изменения $m$ выделяют зону действия соотношения (4.11). Положив

$$
\Lambda(t, s):=-s \ln \theta(1)+(1-s) \psi\left(t(1-s)^{-1}\right), \quad s \in\left[0,1-t\left(\mu^{+}\right)^{-1}\right),
$$

получаем, что слагаемые (4.3) суммы (4.2) эквивалентны выражению:

$$
c_{4}(t, s)(n-n s)^{-1 / 2} e^{-\Lambda(t, s) n}, \quad s:=m n^{-1}, \quad c_{4}(t, s):=c_{1} c_{3}^{t /(1-s)},
$$

равномерно по $a_{n} \leqslant m \leqslant\left(1-t / \mu^{+}-\varepsilon\right) n$, где $a_{n} \rightarrow \infty$ при $n \rightarrow \infty$.

j. Дифференцируем функцию $\Lambda(t, s)$ по переменной $s$ :

$$
\frac{\partial \Lambda(t, s)}{\partial s}=\ln \frac{\theta\left(\lambda\left(t(1-s)^{-1}\right)\right)}{\theta(1)}, \quad \frac{\partial^{2} \Lambda(t, s)}{\partial s^{2}}=\frac{t^{2}}{(1-t)^{3} \sigma^{2}\left(\lambda\left(t(1-s)^{-1}\right)\right)} .
$$

Ввиду монотонности функции $\lambda(\cdot)$ и неравенства $\lambda\left(t(1-s)^{-1}\right)>$ $\lambda(0)>1$ условие $\theta\left(\lambda\left(t(1-s)^{-1}\right)\right)=\theta(1)$ обращения в нуль первой производной приводит к уравнению $t(1-s)^{-1}=\mu^{*}$, решение которого мы обозначим $\widehat{s}_{t}:=1-t / \mu^{*}$. Поскольку вторая производная положительна, то функция $\Lambda(t, s)$ по переменной $s$ имеет единственный минимум в точке $s=\widehat{s}_{t}$, равный $\Lambda\left(t, \widehat{s}_{t}\right)=\widehat{\psi}(t)$ (см. п. 2а). В окрестности точки $s=\widehat{s}_{t}$ запишем разложение Тейлора:

$$
\Lambda\left(t, \widehat{s}_{t}+\Delta s\right)=\widehat{\psi}(t)+\frac{1}{2 b_{t}^{2}} \Delta s^{2}+O\left(\Delta s^{3}\right), \quad b_{t}^{2}:=\frac{t \sigma^{2}\left(\lambda^{*}\right)}{\left(\mu^{*}\right)^{3}} .
$$

k. Пусть $t \in\left(0, \mu^{*}\right)$, так что $\widehat{s}_{t}>0$. Слагаемые суммы (4.2) при $\left|m / n-\widehat{s}_{t}\right| \leqslant n^{-3 / 8}$ заменим их асимптотическими выражениями (4.17), к которым применим разложение Тейлора (4.19), и получим:

$$
\frac{c_{4}\left(t, \widehat{s}_{t}\right) e^{-\hat{\psi}(t) n}}{\sqrt{n\left(1-\widehat{s}_{t}\right)}} \cdot \exp \left\{-\frac{1}{2 b_{t}^{2}}\left(\frac{m}{n}-\widehat{s}_{t}\right)^{2} n+O(1)\left(\frac{m}{n}-\widehat{s}_{t}\right)^{3} n\right\},
$$

где член $O(1)$ равномерно ограничен в указанном диапазоне $m$. Заметив, что $\left|m / n-\widehat{s}_{t}\right|^{3} n \leqslant n^{-1 / 8}$, перейдем к интегральной сумме и получим

$$
\sum_{\left\{\left|m / n-\hat{s}_{t}\right| \leqslant n^{-3 / 8}\right\}} \exp \left\{-\frac{\left(m-n \widehat{s}_{t}\right)^{2}}{2 b_{t}^{2} n}\right\} \sim \sqrt{2 \pi} b_{t} \sqrt{n}, \quad n \rightarrow \infty .
$$

Таким образом, для суммы членов (4.20) имеем асимптотику

$$
c_{5} e^{-\hat{\psi}(t) n}, \quad c_{5}:=c_{4}\left(t, \widehat{s}_{t}\right)\left(1-\widehat{s}_{t}\right)^{-1 / 2} \sqrt{2 \pi} b_{t}=c_{1} \mathbf{P}\left(L_{\infty}^{\left(\mu^{*}\right)}=0\right) \Gamma\left(\lambda^{*}\right)\left(\mu^{*}\right)^{-1} .
$$

Далее мы покажем, что слагаемыми суммы (4.2) за пределами отрезка $\left|m / n-\widehat{s}_{t}\right| \leqslant n^{-3 / 8}$ можно пренебречь, так что асимптотика суммы (4.2) дается выражением (4.21). 
1. На концах отрезка $\left|m / n-\widehat{s}_{t}\right| \leqslant n^{-3 / 8}, t>0$, второй сомножитель в выражении (4.20) эквивалентен величине $\exp \left\{-\left(2 b_{t}^{2}\right)^{-1} n^{1 / 4}\right\}$. Поэтому, ввиду (4.17) и свойств функции $\Lambda(t, s)$ (см. п. $\mathbf{j}$ ), слагаемые суммы (4.2) при $\left|m / n-\widehat{s}_{t}\right|>n^{-3 / 8}, 0<\varepsilon<m / n<1-t / \mu^{+}-\varepsilon$, получают по сравнению с центральными членами дополнительный множитель, экспоненциально малый по $n^{1 / 4}$. Остается рассмотреть области $m \leqslant \varepsilon n$ и $m \geqslant\left(1-t / \mu^{+}-\varepsilon\right) n$ для сколь угодно малого $\varepsilon>0$. При $m \leqslant \varepsilon n$ слагаемые (4.3) оценим сверху, отбросив в (4.3) первый сомножитель, и получим границу:

$$
\mathbf{E}\left(\left(1+e^{-S_{n-m}}\right)^{-N_{t}} ; L_{n-m}=0\right) \leqslant \mathbf{E}\left(\left(1+e^{-S_{n-m}}\right)^{\left[e^{t(n-m)}\right]} ; L_{n-m}=0\right) .
$$

Далее используем (4.11) при $\nu=t$ и получим, что экспоненциальный член асимптотики правой части (4.22) равен $\exp (-\psi(t)(n-m)) \leqslant$ $\exp (-\psi(t)(1-\varepsilon) n)$. Напомним, что $\widehat{\psi}(t)$ представляет касательную к выпуклой вниз функции $\psi(t)$ в точке $t=\mu^{*}$, так что $\psi(t)>\widehat{\psi}(t)$ при $t \neq \mu^{*}$. Полагая, что $\psi(t)(1-\varepsilon)>\widehat{\psi}(t)$, приходим к выводу, что рассматривамые слагаемые суммы (4.2) пренебрежимо малы.

m. Пусть $\varepsilon>0$ настолько мало, что выполнено неравенство $1-\mu^{*} / \mu^{+}-\varepsilon>0$. При $n-1 \geqslant m \geqslant\left(1-t / \mu^{+}-\varepsilon\right) n$ используем оценку (4.5) для первого сомножителя в (4.3), а второй сомножитель оценим сверху выражением:

$$
\mathbf{E}\left(\left(1+e^{-S_{n-m}}\right)^{-N_{t}} ; S_{n-m} \leqslant(t-\varepsilon) n\right)+\mathbf{P}\left(S_{n-m}>(t-\varepsilon) n\right) .
$$

Первым слагаемым в (4.23) мы пренебрегаем, так как оно убывает, как двойная экспонента по $n$ (см. (4.13) и далее). Ко второму слагаемому применим неравенство из п. $2 \mathbf{c}$ :

$\mathbf{P}\left(S_{(1-s) n}>(t-\varepsilon) n\right) \leqslant \exp \left\{-\psi\left(\frac{t-\varepsilon}{1-s} ; \lambda\right)(1-s) n\right\}, \quad s:=\frac{m}{n}, \quad \lambda>\lambda^{*}$.

Оценка сверху для рассматриваемых слагаемых суммы (4.2) получается умножением правой части неравенства на $c_{1} \theta(1)^{m}$ (см. (4.5)). Множитель при $n$ в экспоненте будет равен

$$
\begin{aligned}
& s \ln \theta(1)-\psi\left((t-\varepsilon)(1-s)^{-1} ; \lambda\right)(1-s) \\
& \quad=s \ln \theta(1)+(1-s) \ln \theta(\lambda)-\lambda(t-\varepsilon) \\
& \quad=s(\ln \theta(1)-\ln \theta(\lambda))+\ln \theta(\lambda)-\lambda \cdot(t-\varepsilon) .
\end{aligned}
$$

Так как $\theta(\lambda)>\theta(1)=\theta\left(\lambda^{*}\right)$, то этот множитель убывает по $s$, а его наибольшее значение для $s \geqslant 1-t / \mu^{+}-\varepsilon$ равно

$$
\left(1-t\left(\mu^{+}\right)^{-1}\right) \ln \theta(1)+t\left(\mu^{+}\right)^{-1} \ln \theta(\lambda)+\varepsilon(\ln \theta(\lambda)-\ln \theta(1)+\lambda)-\lambda t .
$$


Вычтем отсюда $(-\widehat{\psi}(t))$ и проведем элементарные преобразования:

$$
t\left(\mu^{+}\right)^{-1}\left[(\ln \theta(\lambda)-\ln \theta(1))-\mu^{+} \cdot\left(\lambda-\lambda^{*}\right)\right]+\varepsilon(\ln \theta(\lambda)-\ln \theta(1)+\lambda) .
$$

Ввиду выпуклости функции $\ln \theta(\lambda)$ имеем неравенство

$$
\ln \theta(\lambda)-\ln \theta\left(\lambda^{*}\right)<\mu^{*} \cdot\left(\lambda-\lambda^{*}\right)<\mu^{+} \cdot\left(\lambda-\lambda^{*}\right),
$$

так что выражение в квадратных скобках в (4.24) отрицательно. Для $t \in[\alpha, \beta] \subseteq\left(0, \mu^{+}\right)$все выражение (4.24) будет отрицательным при достаточно малом $\varepsilon>0$, откуда следует, что рассматриваемые слагаемые не вносят вклада в асимптотику суммы (4.2). Остается заметить, что слагаемое суммы (4.2), соответствующее $m=n$, меньше $2^{-N_{t}}$.

n. Из оценок пп. k, l, m, b следует, что

$$
\mathbf{E}\left(e^{L_{n}}\left(1+e^{L_{n-1}-S_{n}}\right)^{-N_{t}}\right) \sim \mathbf{E}\left(e^{L_{n}}\left(1+e^{L_{n}-S_{n}}\right)^{-N_{t}}\right) \sim c_{5} e^{-\hat{\psi}(t) n}
$$

при $n \rightarrow \infty$ равномерно по $t \in[\alpha, \beta] \subseteq\left(0, \mu^{*}\right)$. Незначительная модификация проведенных рассуждений приводит к равномерному по $t$ соотношению:

$$
\mathbf{E}\left(e^{L_{n}}\left(1+n^{-1} e^{L_{n}-S_{n}}\right)^{-N_{t}}\right) \sim c_{5} n^{\lambda_{t}} e^{-\hat{\psi}(t) n}, \quad n \rightarrow \infty .
$$

Именно, математическое ожидание в (4.26) разлагаем в сумму вида (4.2). Второй сомножитель в представлении типа (4.3) оцениваем с помощью асимптотики (4.15). В результате, суммируя по $m$ таким, что $\mid m / n-$ $\widehat{s}_{t} \mid \leqslant n^{-3 / 8}$ (см. п. $\mathbf{k}$ ), вместо (4.21) получаем правую часть (4.26). K оценке п. $\mathbf{k}$ добавляется степенной множитель $(n-m)^{\lambda_{t}}$. Оценки пп. $\mathbf{l}, \mathbf{m}$ сохраняются.

Заключение теоремь 1 для $t \in\left[\varepsilon, \mu^{*}-\varepsilon\right)$ следует из соотношений (4.1), (4.25), (4.26).

о. При $t=\mu^{*}$ функция $\Lambda(t, s)$ достигает максимума $\widehat{\psi}\left(\mu^{*}\right) \equiv \psi\left(\mu^{*}\right)$ в точке $s=0$ (см. п. $\mathbf{j}$ ). Соответственно, область суммирования в п. (4.2) разбиваем на части $m / n \leqslant n^{-3 / 8}$ и $m / n>n^{-3 / 8}$. Ввиду (4.11) имеем при $n \rightarrow \infty$ :

$$
\mathbf{E}\left(\left(1+e^{-S_{n-m}}\right)^{-N_{\mu^{*}}} ; L_{n-m}=0\right) \sim \frac{c_{3}^{\left(\mu^{*}\right)}}{\sqrt{n-m}} \exp \left\{-\psi\left(\frac{\mu^{*} n}{n-m}\right)(n-m)\right\}
$$

равномерно по $m \leqslant\left(1-\mu^{*} / \mu^{+}-\varepsilon\right) n, \varepsilon>0$. По формуле Тейлора имеем:

$$
\psi\left(\frac{\mu^{*} n}{n-m}\right)=\psi\left(\mu^{*}\right)+\lambda^{*} \mu^{*} \frac{m}{n-m}+\frac{1}{2 b_{\mu^{*}}^{2}}\left(\frac{m}{n-m}\right)^{2}+O(1)\left(\frac{m}{n}\right)^{3}
$$

при $m \leqslant n^{5 / 8}, b_{\mu^{*}}^{2}=\sigma^{2}\left(\lambda^{*}\right) /\left(\mu^{*}\right)^{2}$ (см. определение $b_{t}^{2}$ в (4.19)). После подстановки (4.28) в (4.27) получаем для суммы по $m \leqslant n^{5 / 8}$ эквивалентное 
выражение:

$$
\frac{c_{3}^{\left(\mu^{*}\right)}}{\sqrt{n}} e^{-\psi\left(\mu^{*}\right) n} \sum_{m=0}^{\left[n^{5 / 8}\right]} \mathbf{E}\left(e^{L_{m}} ; K_{m}=m\right) \exp \left\{-m \ln \theta(1)-\frac{1}{2 b_{\mu^{*}}^{2}} \frac{m^{2}}{n-m}\right\}
$$

Используя соотношение (4.5), выводим, что сумма в (4.29) эквивалентна $c_{1} \sqrt{\pi / 2} b_{\mu^{*}} \sqrt{n}$. Таким образом, все выражение (4.29) эквивалентно выражению

$$
c_{6} \exp \left(-\psi\left(\mu^{*}\right) n\right), \quad c_{6}:=c_{1}\left(2 \mu^{*}\right)^{-1} \mathbf{P}\left(L_{\infty}^{\left(\mu^{*}\right)}=0\right) \Gamma\left(\lambda^{*}\right)=c_{5} 2^{-1} .
$$

р. Проверим, что при $t=\mu^{*}$ часть суммы $(4.2)$ по $m / n>n^{-3 / 8}$ пренебрежимо мала, так что выражение (4.2) при $t=\mu^{*}$ имеет асимптотику (4.30). Члены этой суммы при $m / n \leqslant 1-\mu^{*} / \mu^{+}-\varepsilon, \varepsilon>0$, эквивалентны при $n \rightarrow \infty$ следующему выражению (см. (4.27), (4.5)):

$$
c_{1} c_{3}^{\left(\mu^{*}\right)}(n-m)^{-1 / 2} \exp \left\{\left(\frac{m}{n} \ln \theta(1)-\psi\left(\frac{\mu^{*} n}{n-m}\right) \frac{n-m}{n}\right) n\right\}
$$

равномерно по указанным $m$. Под знаком экспоненты в $(4.31)$ стоит величина $-\Lambda\left(\mu^{*}, m / n\right) n$ (см. (4.16)). Поскольку функция $\Lambda\left(\mu^{*}, s\right), s \geqslant 0$, строго возрастает, то $\Lambda\left(\mu^{*}, m / n\right) \geqslant \Lambda\left(\mu^{*},\left[n^{5 / 8}\right] / n\right)$ при $m>n^{5 / 8}$. По формуле Тейлора (см. (4.19)) получаем, что $\Lambda\left(\mu^{*}, n^{-3 / 8}\right)=\psi\left(\mu^{*}\right)+(1+$ $o(1))\left(2 b_{\mu^{*}}^{2}\right)^{-1} n^{-3 / 4}$ и, следовательно, экспонента в (4.31) мажорируется величиной $(4.30)$, умноженной на $c e^{-\gamma n^{1 / 4}}$ при некоторых $c, \gamma>0$. Следовательно, часть второй суммы при $n^{5 / 8}<m \leqslant\left(1-\mu^{*} / \mu^{+}-\varepsilon\right) n$ пренебрежимо мала. Для остатка суммы по $m>\left(1-\mu^{*} / \mu^{+}-\varepsilon\right) n$ сохраняется оценка п. $\mathbf{m}$.

r. Рассуждения пп. о, p приводят к асимптотике (4.25) при $t=\mu^{*}$, в которой $c_{5}$ заменяется на $c_{6}$ (см. (4.30)). Это дает нижнюю оценку для вероятностей больших уклонений. Элементарная модификация рассуждений п. $\mathbf{n}$ приводит к соотношению (4.26) при $t=\mu^{*}$ и $c_{5}^{(t)}$, замененном на $c_{6}$. Отсюда следует утверждение теоремы 1 при $t=\mu^{*}$.

s. Пусть последовательность $t_{n} \in\left[\mu^{*}-\varepsilon, \mu^{*}\left(1-n^{-3 / 8}\right)\right]$ произвольна. Покажем, что неравенства (3.1) с заменой $t$ на $t_{n}$ остаются верными. Во-первых, отметим, что асимптотика (4.11) остается справедливой и в случае замены $t$ на $t_{n}$, при этом сохраняется равномерность по всем таким последовательностям $t_{n}$. В сумме (4.2) выделяем часть $\mid m / n-$ $\widehat{s}_{t_{n}} \mid \leqslant n^{-3 / 8}$. Записав разложение Тейлора, находим (ср. (4.28), (4.20)):

$$
\psi\left(\frac{t_{n} n}{n-m}\right)=\psi\left(\mu^{*}\right)+\lambda \cdot\left(\frac{t_{n} n}{n-m}-\mu^{*}\right)+\frac{1}{2 b_{\mu^{*}}^{2}} \frac{\left(m-\widehat{s}_{t_{n}} n\right)^{2}}{(n-m)^{2}}+\frac{O(1)}{n^{9 / 8}}
$$

После элементарных выкладок, как и в п. $\mathbf{k}$, приходим к асимптотике (4.21) при $t=t_{n}$. Дальнейший анализ подобен проведенному выше 
для случая $t \in\left[\alpha, \mu^{*}-\varepsilon\right]$ и приводит к заключению теоремы 1 для указанных $t$.

t. Пусть $t_{n} \in\left[\mu^{*}\left(1-n^{-3 / 8}\right), \mu^{*}\right]$. В этом случае также выписываем разложение Тейлора (4.32) для $m \leqslant \widehat{s}_{t_{n}} n+n^{5 / 8}$. Далее имеем (см. пп. $\mathbf{k}, \mathbf{o})$ :

$$
\sum_{m \leqslant \hat{s}_{t_{n}} n+n^{5 / 8}} \exp \left\{-\frac{\left(m-n \widehat{s}_{t_{n}}\right)^{2}}{2 b_{\mu^{*}}^{2} n}\right\} \sim c_{5} \Phi\left(\frac{\sqrt{n} \widehat{s}_{t_{n}}}{b_{\mu^{*}}}\right) e^{-\hat{\psi}\left(t_{n}\right)}, \quad n \rightarrow \infty
$$

где $\Phi(\cdot)$ - функция распределения стандартного нормального закона. Как и выше, устанавливается, что правая часть в (4.33) дает главный член асимптотики всей суммы. Асимптотика нижней оценки вероятностей больших уклонений выводится тем же способом, что и для $t \in\left[\alpha, \mu^{*}-\varepsilon\right]$.

5. Доказательство теоремы 2. Положим $M_{n}:=\max _{0 \leqslant j \leqslant n} S_{j}$, $M_{\infty}:=\max _{j \geqslant 0} S_{j}, T_{n}:=\min \left(j \leqslant n: S_{j}=M_{n}\right), L_{1 m}:=\min _{1 \leqslant j \leqslant m} S_{j}$ и при $t \in\left(0, \mu^{+}\right]$введем сл.в. $L_{1 n}^{(t)}:=\min _{1 \leqslant j \leqslant n} S_{j}^{(t)}, L_{1 \infty}^{(t)}:=\min _{j \geqslant 1} S_{j}^{(t)}$. Представим математическое ожидание $\mathbf{E} e^{\kappa M_{n}}$ в виде суммы по разбиению $\left\{T_{n}=m\right\}, m=0,1, \ldots, n$. Замечая, что $\left\{T_{n}=m\right\}=\left\{T_{m}=m\right.$, $S_{j}-S_{m} \leqslant 0$ при $\left.m \leqslant j \leqslant n\right\}$, получаем:

$$
\mathbf{E} e^{\kappa M_{n}}=\sum_{m=0}^{n} \mathbf{E}\left(e^{\kappa M_{m}} ; T_{m}=m\right) \mathbf{P}\left(M_{n-m}=0\right)
$$

Переходя к обращенному случайному блужданию, находим:

$$
\begin{aligned}
\mathbf{E}\left(e^{\kappa M_{m}} ; T_{m}=m\right) & =\mathbf{E}\left(e^{\kappa S_{m}} ; T_{m}=m\right)=\mathbf{E}\left(e^{\kappa S_{m}} ; L_{1 m}>0\right) \\
& =\int_{\left\{l_{1 m}>0\right\}} \ldots \int^{\kappa s_{m}} \prod_{i=1}^{m} d F\left(x_{i}\right) \\
& =\int_{\left\{l_{1 m}>0\right\}} \ldots \int_{i=1}^{m} d F^{\left(\mu_{\kappa}\right)}\left(x_{i}\right)=\mathbf{P}\left(L_{1 m}^{\left(\mu_{\kappa}\right)}>0\right),
\end{aligned}
$$

где $l_{1 m}:=\min _{1 \leqslant j \leqslant m} s_{j}, s_{j}=\sum_{i=1}^{j} x_{i}$. Подставляя (5.2) в (5.1) и учитывая, что

$$
\begin{aligned}
\mathbf{P}\left(L_{1 m}^{\left(\mu_{\kappa}\right)}>0\right) & \rightarrow \mathbf{P}\left(L_{1 \infty}^{\left(\mu_{\kappa}\right)}>0\right) \quad \text { при } \quad m \rightarrow \infty, \\
\mathbf{P}\left(M_{n-m}=0\right) & \rightarrow \mathbf{P}\left(M_{\infty}=0\right) \quad \text { при } \quad n-m \rightarrow \infty,
\end{aligned}
$$

приходим к заключению теоремы с $C_{2}=\mathbf{P}\left(L_{1 \infty}^{\left(\mu_{\kappa}\right)}>0\right) \mathbf{P}\left(M_{\infty}=0\right)$. 
6. Доказательство теоремы 3.

a. Используя обозначения из п. 5, представим математическое ожидание $\mathbf{E} U_{n}^{\kappa}$ в следующем виде:

$$
\sum_{m=0}^{n} \mathbf{E}\left\{e^{\kappa M_{m}}\left(\sum_{j=1}^{m-1} e^{S_{j}-M_{m}}+\sum_{j=m}^{n} e^{S_{j}-M_{m}}\right)^{\kappa} ; T_{n}=m\right\} .
$$

Введем экземпляр $\widetilde{S}_{j}$ случайного блуждания $S_{j}$, независимый от $S_{j}$. Для обозначения функционалов от блуждания $\widetilde{S}_{j}$ будем употреблять те же буквы, что и в случае блуждания $S_{j}$, снабжая их «волной».

Представим слагаемые суммы (6.1) в виде

$$
\begin{gathered}
\mathbf{E}\left\{e^{\kappa S_{m}}\left(V_{m}+\widetilde{U}_{n-m}\right)^{\kappa} ; L_{1 m}>0, \widetilde{M}_{n-m}=0\right\}, \\
L_{1 m}=\min _{1 \leqslant j \leqslant m} S_{j}, \quad V_{m}:=\sum_{j=1}^{m} e^{-S_{j}}, \quad \widetilde{U}_{k}:=\sum_{j=0}^{k} e^{\widetilde{S}_{j}}, \quad \widetilde{M}_{k}:=\max _{o \leqslant j \leqslant k} \widetilde{S}_{j} .
\end{gathered}
$$

Положим $v_{m}:=\sum_{j=1}^{m} e^{-s_{j}}, u_{k}:=\sum_{j=0}^{k} e^{s_{j}}, \bar{s}_{k}:=\max _{1 \leqslant j \leqslant k} s_{j}$,

$$
J_{k}(v):=\int_{\left\{\bar{s}_{k} \leqslant 0\right\}} \ldots \int_{0}\left(v+u_{k}\right)^{\kappa} \prod_{i=1}^{k} d F\left(x_{i}\right)=\mathbf{E}\left(\left(v+U_{k}\right)^{\kappa} ; M_{k}=0\right), \quad v \geqslant 0,
$$

и представим математическое ожидание (6.2) в виде

$$
\mathbf{E}\left(e^{\kappa S_{m}} J_{n-m}\left(V_{m}\right) ; L_{1 m}>0\right)=\int_{\left\{l_{1 m}>0\right\}} \ldots \int_{n-m}\left(v_{m}\right) \prod_{i=1}^{m} d F^{\left(\mu_{\kappa}\right)}\left(x_{i}\right),
$$

где использованы обозначения $s_{j}=\sum_{i=1}^{j} x_{i}, l_{1 m}=\min _{1 \leqslant i \leqslant m} s_{i}$ и учтено, что $e^{\kappa x} d F(x)=\theta(\kappa) d F^{\left(\mu_{\kappa}\right)}(x), \theta(\kappa)=1$.

b. Покажем, что при $k \rightarrow \infty$

$$
J_{k}(v) \rightarrow J_{\infty}(v):=\mathbf{E}\left(\left(v+U_{\infty}\right)^{\kappa} ; M_{\infty}=0\right), \quad U_{\infty}:=\sum_{j=0}^{\infty} e^{S_{j}},
$$

равномерно на ограниченных множествах, функции $J_{k}(v)$ непрерывны.

Пусть $0<\delta<1, \mu_{0}<\gamma<0$, и предположим для упрощения записи, что число $\delta x$ целое. Представим вероятность $\mathbf{P}\left(U_{k}>x, M_{k}=0\right), x \geqslant 1$, в виде суммы по разбиению из трех событий

$$
\left\{S_{\delta x}>2^{-1} \mu_{0} \delta x\right\}, \quad\left\{S_{\delta x} \leqslant 2^{-1} \mu_{0} \delta x, \mathscr{B}\right\}, \quad\left\{S_{\delta x} \leqslant 2^{-1} \mu_{0} \delta x, \overline{\mathscr{B}}\right\},
$$

где $\mathscr{B}:=\left\{S_{j} \leqslant \gamma(j-\delta x), \delta x \leqslant j \leqslant k\right\}$. На множестве $\mathscr{B} \cap\left\{M_{k}=0\right\}$ выполняется неравенство

$$
U_{k} \leqslant \delta x+\sum_{j=\delta x}^{k} e^{\gamma(j-\delta x)} \leqslant \delta x+\left(1-e^{\gamma}\right)^{-1} .
$$


Тем самым, пересечение $\left\{U_{k}>x\right\} \cap \mathscr{B} \cap\left\{M_{k}=0\right\}$ пусто для достаточно больших $x$. Далее, имеем:

$$
\begin{aligned}
\mathbf{P}\left(S_{\delta x}>2^{-1} \mu_{0} \delta x\right) & \leqslant \exp \left(-\psi\left(2^{-1} \mu_{0}\right) \delta x\right) \\
\mathbf{P}\left(S_{\delta x} \leqslant 2^{-1} \mu_{0} \delta x, \overline{\mathscr{B}}\right) & =\mathbf{P}\left(\bigcup_{j=\delta x}^{k}\left\{S_{j}>\gamma(j-\delta x), S_{\delta x} \leqslant \frac{1}{2} \pi \mu_{0} \delta x\right\}\right) \\
& \leqslant \mathbf{P}\left(\max _{i \geqslant 1}\left(S_{i}-\gamma i\right)>-\frac{1}{2} \mu_{0} \delta x\right),
\end{aligned}
$$

где учтено, что на множестве $\left\{S_{\delta x} \leqslant \mu_{0} \delta x / 2\right\}$ имеет место включение

$$
\left\{S_{j}>\gamma(j-\delta x)\right\} \subseteq\left\{S_{j}-S_{\delta x}>\gamma(j-\delta x)-2^{-1} \mu_{0} \delta x\right\} .
$$

Используя оценку из п. $2 \mathbf{d}$, в итоге получаем для достаточно больших $x$ и всех $k$ :

$$
\mathbf{P}\left(U_{k}>x, M_{k}=0\right) \leqslant \exp \left(-\psi\left(2^{-1} \mu_{0}\right) \delta x\right)+\exp \left(-2^{-1} \mu_{0} q_{\gamma} \delta x\right),
$$

где $q_{\gamma}>0$ есть решение уравнения $e^{-\lambda \gamma} \theta(\lambda)=1$.

Из монотонной сходимости $U_{k} \rightarrow U_{\infty}$ п.н., $M_{k} \rightarrow M_{\infty}$ п.н. при $k \rightarrow$ $\infty$ следует, что последовательность функций распределения $\mathbf{P}\left(U_{k} \leqslant x\right.$, $\left.M_{k}=0\right)$ слабо сходится к функции распределения $\mathbf{P}\left(U_{\infty} \leqslant x, M_{\infty}=0\right)$. С учетом неравенства (6.6) получаем, что меры $x^{\kappa} \mathbf{P}\left(U_{k} \leqslant x, M_{k}=0\right)$ слабо сходятся к конечной мере $x^{\kappa} \mathbf{P}\left(U_{\infty} \leqslant x, M_{k}=0\right)$.

Представим функцию $J_{k}(v)$ в виде

$$
\int_{1}^{A}(v+x)^{\kappa} d \mathbf{P}\left(U_{k} \leqslant x, M_{k}=0\right)+\int_{A+}^{\infty}(v+x)^{\kappa} d \mathbf{P}\left(U_{k} \leqslant x, M_{k}=0\right) .
$$

Для $\kappa>1$ производная $\kappa(v+x)^{\kappa-1}$ при $x \in[1, A], v \in[0, B]$ не превосходит $\kappa(A+B)^{\kappa-1}$. Отсюда легко следует, что первый интеграл в (6.7) непрерывен по $v$, а его сходимость к $\int_{1}^{A}(v+x)^{\kappa} d \mathbf{P}\left(U_{\infty} \leqslant x, M_{\infty}=0\right)$ в случае, когда $A$ есть точка непрерывности предельной меры, равномерна по $v \in[0, B]$. Второй интеграл в (6.7) может быть сделан ввиду (6.6) сколь угодно малым за счет выбора $A$. Это верно также для интеграла по мере $\mathbf{P}\left(U_{\infty} \leqslant x, M_{\infty}=0\right)$, и соотношение (6.4) установлено.

c. Интеграл (6.3) перепишем в виде

$$
\mathbf{E}\left(J_{n-m}\left(V_{m}^{\left(\mu_{\kappa}\right)}\right) ; L_{1 m}^{\left(\mu_{\kappa}\right)}>0\right)=\int_{0}^{\infty} J_{n-m}(v) d \mathbf{P}\left(V_{m}^{\left(\mu_{\kappa}\right)} \leqslant v, L_{1 m}^{\left(\mu_{\kappa}\right)}>0\right),
$$

где $V_{m}^{(\nu)}:=\sum_{j=1}^{m} e^{-S_{j}^{(\nu)}}, L_{1 m}^{(\nu)}=\min _{1 \leqslant j \leqslant m} S_{j}^{(\nu)}$. Рассуждения, подобные проведенным в предыдущем пункте, приводят к оценке (ср. (6.6))

$$
\mathbf{P}\left(V_{m}^{\left(\mu_{\kappa}\right)}>v ; L_{1 m}^{\left(\mu_{\kappa}\right)}>0\right) \leqslant \exp \left(-\psi^{\left(\mu_{\kappa}\right)}\left(2^{-1} \mu_{\kappa}\right) \delta v\right)+\exp \left(-2^{-1} \mu_{\kappa} q^{\left(\mu_{\kappa}\right)} \delta v\right) .
$$


Область интегрирования в (6.8) разобьем на части $[0, B]$ и $(B, \infty)$. Из элементарного неравенства $(v+u)^{\kappa} \leqslant 2^{\kappa}\left(v^{\kappa}+u^{\kappa}\right), u, v \geqslant 0$, получаем: $J_{k}(v) \leqslant 2^{\kappa} v^{\kappa}+2^{\kappa} J_{k}(0)$. Отсюда и из оценки (6.9) вытекает, что интеграл $(6.8)$ по области $(B, \infty)$ может быть сделан за счет выбора $B$ сколь угодно малым равномерно по $n$ и $m \leqslant n$. В интеграле (6.8) по области $[0, B]$ перейдем к пределу при $n \rightarrow \infty, b_{n} \leqslant m \leqslant n-b_{n}, b_{n} \rightarrow \infty, b_{n}=o(n)$. Принимая во внимание равномерную на $[0, B]$ сходимость $J_{k}(v) \rightarrow J_{\infty}(v)$ и слабую сходимость функций распределения $\mathbf{P}\left(V_{m}^{\left(\mu_{\kappa}\right)} \leqslant v, L_{1 m}^{\left(\mu_{\kappa}\right)}>0\right)$ к функции распределения $\mathbf{P}\left(V_{\infty}^{\left(\mu_{\kappa}\right)} \leqslant v, L_{1 \infty}^{\left(\mu_{\kappa}\right)}>0\right)$, приходим к заключению, что математическое ожидание (6.8) (а вместе с ним и (6.2)) сходится при $n \rightarrow \infty$ равномерно по $b_{n} \leqslant m \leqslant n-b_{n}, b_{n} \rightarrow \infty, b_{n}=o(n)$ к математическому ожиданию

$$
\mathbf{E}\left(J_{\infty}\left(V_{\infty}^{\left(\mu_{\kappa}\right)}\right) ; L_{1 \infty}^{\left(\mu_{\kappa}\right)}>0\right)=\mathbf{E}\left(\left(V_{\infty}^{\left(\mu_{\kappa}\right)}+\widetilde{U}_{\infty}\right)^{\kappa} ; L_{1 \infty}^{\left(\mu_{\kappa}\right)}>0, \widetilde{M}_{\infty}=0\right)=: C_{3} .
$$

Отсюда следует заключение теоремы 3.

\section{7. Доказательство теорем 4, 5.} (cp. (1.1))

а. Положим $\check{U}_{k}:=\sum_{j=0}^{k} e^{S_{n}-S_{j}}$. В математическом ожидании

$$
\mathbf{E}\left\{\left(1+V_{n}\right)^{-1}\left(1+\check{U}_{n-1}^{-1}\right)^{-N_{t}}\right\} \equiv \mathbf{P}\left(\ln Z_{n}>t n\right), \quad V_{n}=\sum_{j=1}^{n} e^{-S_{j}}
$$

заменим $\check{U}_{n-1}^{-1}$ на $\check{U}_{n}^{-1}$ и покажем, что происходящее при этом увеличение пренебрежимо мало при $n \rightarrow \infty$ по сравнению с асимптотикой (3.2). В случае $\check{U}_{n} \leqslant e^{3 n t / 4}$ выражения $\left(1+\check{U}_{n-1}^{-1}\right)^{-N_{t}}$ и $\left(1+\check{U}_{n}^{-1}\right)^{-N_{t}}$ убывают как двойная экспонента по $n$, а потому, ввиду теоремы 1 , достаточно провести сравнение математических ожиданий, суженных на область $\check{U}_{n}>e^{3 n t / 4}$. Представим значения сл.в. $\check{U}_{n}$ в форме $e^{x n}, x>3 t / 4$, учтем, что $\check{U}_{n}=1+\check{U}_{n-1}$, и, переходя к логарифмам, получим:

$$
\begin{aligned}
-N_{t} \ln \left(1+\left(-1+e^{x n}\right)^{-1}\right) & =-N_{t}\left(e^{-x n}\left(1-e^{-x n}\right)^{-1}+O\left(e^{-3 n t / 2}\right)\right) \\
& =-N_{t} e^{-x n}+O\left(e^{-n t / 2}\right), \\
-N_{t} \ln \left(1+e^{-x n}\right) & =-N_{t} e^{-x n}+O\left(e^{-n t / 2}\right) .
\end{aligned}
$$

Отсюда следует, что различие математических ожиданий, суженных на область $\check{U}_{n}>e^{3 n t / 4}$, сводится к дополнительному множителю $e^{O\left(e^{-n t / 2}\right)}=$ $1+O\left(e^{-n t / 2}\right)$, которым можно пренебречь. Таким образом, для доказательства теорем 4,5 достаточно исследовать асимптотику математического ожидания

$$
\mathbf{E}\left\{\left(1+V_{n}\right)^{-1}\left(1+\check{U}_{n}^{-1}\right)^{-N_{t}}\right\} \equiv \mathbf{E}\left\{\left(1+V_{n}\right)^{-1}\left(1+e^{-S_{n}}\left(1+V_{n}\right)^{-1}\right)^{-N_{t}}\right\} .
$$


Как и в п. 4, мы представим (7.1) в виде суммы математических ожиданий по разбиению $\left\{K_{n}=m\right\}, m=0,1, \ldots, n$ (cp. (4.2)). Сумма по $\left|m-n \widehat{s}_{t}\right|>n^{5 / 8}$, как следует из п. 4 , пренебрежимо мала по сравнению с $e^{-\hat{\psi}(t) n}$. Поэтому далее исследуется сумма по $\left|m-n \widehat{s}_{t}\right| \leqslant n^{5 / 8}$.

Воспользуемся тем, что в области $\left\{(x, v): e^{-x}(1+v)^{-1} \leqslant e^{\delta n},-\infty<\right.$ $x<\infty, v \geqslant 0\}$ для $\delta \in(0, t / 2)$ соотношение

$$
\left(1+e^{-x-t n}(1+v)^{-1}\right)^{-N_{t}} \sim \exp \left(-e^{-x}(1+v)^{-1}\right), \quad n \rightarrow \infty,
$$

выполняется равномерно по $x, v$, а на дополнении к указанной области обе стороны соотношения (7.2) убывают как двойная экспонента от $n$ (см. (4.13)). С учетом теоремы 1 отсюда следует, что математическое ожидание (7.1) имеет ту же асимптотику, что и математическое ожидание

$$
\sum_{\left|m-n \hat{s}_{t}\right| \leqslant n^{5 / 8}}\left\{\left(1+V_{n}\right)^{-1} \exp \left(-e^{-\left(S_{n}-t n\right)}\left(1+V_{n}\right)^{-1}\right) ; K_{n}=m\right\}=: J_{n}^{(t)} .
$$

b. Переходим к исследованию вероятностей больших уклонений $\mathbf{P}\left(\ln Z_{n}>\mu^{*} n\right)$ и заменим их суммой (7.3) при $t=\mu^{*}$. При заданном $m$ воспользуемся представлением

$$
1+V_{n} \stackrel{d}{=} e^{-S_{m}}\left(U_{m}+\widetilde{V}_{n-m}\right), \quad U_{n}=\sum_{j=0}^{n} e^{S_{j}}, \quad \widetilde{V}_{k}:=\sum_{j=1}^{k} e^{-\widetilde{S}_{j}}, \quad \widetilde{V}_{0}:=0,
$$

где $\widetilde{S}_{j}$ - экземпляр случайного блуждания $S_{j}$, от него не зависящий; символ $\stackrel{d}{=}$ означает равенство случайных величин по распределению. В результате $J_{n}^{\left(\mu^{*}\right)}$ представляется в виде

$$
\sum_{m \leqslant n^{5 / 8}} \mathbf{E}\left\{e^{S_{m}}\left(U_{m}+\widetilde{V}_{n-m}\right)^{-1} \exp \left(-e^{-\widetilde{S}_{n-m}+\mu^{*} n}\left(U_{m}+\widetilde{V}_{n-m}\right)^{-1}\right) ; K_{n}=m\right\} .
$$

При $u>1, v>0, m<n$ рассмотрим интеграл

$$
\iint \frac{1}{u+v} \exp \left(-\frac{e^{-x}}{u+v}\right) \mathbf{P}\left(S_{n-m}-\mu^{*} n \in d x, V_{n-m} \in d v ; L_{n-m}=0\right) .
$$

Сделаем в (7.5) замену меры из п. $2 \mathbf{e}$ при $\nu=\mu^{*}$ и запишем результат в виде произведения $\exp \left(-\psi\left(\mu^{*}\right)(n-m)-\lambda^{*} \mu^{*} m\right)$ на интеграл (см. п. 2е)

$$
\begin{aligned}
& J_{n, m}(u):=\iint(u+v)^{-1} \exp \left(-\lambda^{*} x-e^{-x}(u+v)^{-1}\right) \\
& \times \mathbf{P}\left(S_{n-m}^{\left(\mu^{*}\right)}-\mu^{*} n \in d x, V_{n-m}^{\left(\mu^{*}\right)} \in d v ; L_{n-m}^{\left(\mu^{*}\right)}=0\right), \\
& V_{k}^{(\nu)}=\sum_{j=1}^{k} e^{-S_{j}^{(\nu)}}, \quad L_{k}^{(\nu)}=\min _{0 \leqslant j \leqslant k} S_{j}^{(\nu)} .
\end{aligned}
$$


Заметив, что $\left\{K_{n}=m\right\}=\left\{K_{m}=m\right\} \cap\left\{\widetilde{L}_{n-m}=0\right\}, \widetilde{L}_{k}:=\min _{0 \leqslant i \leqslant k} \widetilde{S}_{i}$, запишем математическое ожидание в $(7.4)$ при $1 \leqslant m \leqslant n$ в виде

$$
e^{-\psi\left(\mu^{*}\right) n} \theta\left(\lambda^{*}\right)^{-m} \iint e^{y} J_{n, m}(u) \mathbf{P}\left(S_{m} \in d y, U_{m} \in d u ; S_{j}<0,1 \leqslant j \leqslant m\right) .
$$

c. Мы докажем, что для любого $\Delta>0$

$$
\begin{aligned}
& \mathbf{P}\left(S_{n}^{(\nu)}-\nu(n+k) \in[x, x+\Delta], V_{n}^{(\nu)} \leqslant v, L_{n}^{(\nu)}=0\right) \\
& \quad \sim \Delta\left(\sigma\left(\lambda_{\nu}\right) \sqrt{2 \pi n}\right)^{-1} \exp \left\{-\frac{1}{2}\left(\frac{\nu k}{\sigma\left(\lambda_{\nu}\right) \sqrt{n}}\right)^{2}\right\} \mathbf{P}\left(V_{\infty}^{(\nu)} \leqslant v, L_{\infty}^{(\nu)}=0\right)
\end{aligned}
$$

в каждой точке непрерывности функции распределения $\mathbf{P}\left(V_{\infty}^{(\nu)} \leqslant v\right.$, $\left.L_{\infty}^{(\nu)}=0\right)$ равномерно по $x=O\left(n^{1 / 4}\right), k=O\left(n^{5 / 8}\right), n \rightarrow \infty$. Соотношение (7.8) потребуется для перехода к пределу под знаком интеграла (7.6). Положим для краткости

$$
\mu_{n}^{(\nu)}(d x, d v):=\mathbf{P}\left(S_{n}^{(\nu)}-\nu n \in d x, V_{n}^{(\nu)} \in d v ; L_{n}^{(\nu)}=0\right) .
$$

Оценку сверху в (7.8) получим, заменив событие $\left\{V_{n}^{(\nu)} \leqslant v\right\}$ на $\left\{V_{l_{n}}^{(\nu)} \leqslant v\right\}$ при $l_{n}:=[\sqrt[3]{n}]$, а событие $\left\{L_{n}^{(\nu)}=0\right\}-$ на $\left\{L_{l_{n}}^{(\nu)}=0\right\}$ и разбивая блуждание $S_{j}^{(\nu)}$ на участки $j \leqslant l_{n}$ и $j \in\left[l_{n}+1, n\right]$. Результат представим в виде

$$
\int_{-\infty}^{\infty} \mathbf{P}\left(S_{m_{n}}^{(\nu)}-\nu\left(m_{n}+k\right)+y \in[x, x+\Delta]\right) \mu_{l_{n}}^{(\nu)}(d y ;[0, v])=: I_{n},
$$

где $m_{n}:=n-l_{n}$. Разобьем область интегрирования в (7.9) на части $|y| \leqslant \sqrt[3]{n}$ и $|y|>\sqrt[3]{n}$ и обозначим соответствующие интегралы $I_{1 n}$ и $I_{2 n}$. Подынтегральную вероятность в (7.9) заменим асимптотически эквивалентным выражением из п. 2g. Учитывая, что $x=O\left(n^{1 / 4}\right), k=O\left(n^{5 / 8}\right)$, получим при $|y| \leqslant \sqrt[3]{n}$ :

$$
\begin{gathered}
\left(\frac{x+\nu k-y}{\sigma\left(\lambda_{\nu}\right) \sqrt{m_{n}}}\right)^{2}=\left(\frac{\nu k}{\sigma\left(\lambda_{\nu}\right) \sqrt{n}}\right)^{2}+O\left(n^{-1 / 24}\right) \\
I_{1 n}=\Delta\left(\sigma\left(\lambda_{\nu}\right) \sqrt{2 \pi n}\right)^{-1} \exp \left\{-\frac{1}{2}\left(\frac{\nu k}{\sigma\left(\lambda_{\nu}\right) \sqrt{m_{n}}}\right)^{2}\right\} \mu_{l_{n}}^{(\nu)}([-\sqrt[3]{n}, \sqrt[3]{n}],[0, v]) \\
+o\left(n^{-1 / 2}\right)
\end{gathered}
$$

где член $o\left(n^{-1 / 2}\right)$ равномерно мал по $k$. Устремляя $n$ к $\infty$ и учитывая, что последовательность событий $\left\{V_{n}^{(\nu)} \leqslant v, L_{n}^{(\nu)}=0\right\}$ убывает, получаем (при всех $v$ ):

$$
\lim _{n \rightarrow \infty} \sigma\left(\lambda_{\nu}\right) \sqrt{2 \pi n} \exp \left\{\frac{1}{2}\left(\frac{\nu k}{\sigma\left(\lambda_{\nu}\right) \sqrt{n}}\right)^{2}\right\} I_{1 n}=\Delta \mathbf{P}\left(V_{\infty}^{(\nu)} \leqslant v, L_{\infty}^{(\nu)}=0\right) .
$$


Заметим, что $I_{2 n} \leqslant \mu_{l_{n}}^{(\nu)}(\overline{[-\sqrt[3]{n}, \sqrt[3]{n}]},[1, \infty))=\mathbf{P}\left(\left|S_{l_{n}}^{(\nu)}-\nu l_{n}\right|>\sqrt[3]{n}\right)$. Так как сл.в. $X^{(\nu)}$ при $\nu \in\left(0, \mu^{+}\right)$удовлетворяет двустороннему условию Крамера, то (см. п. 2c):

$\mathbf{P}\left(S_{l_{n}}^{(\nu)}-\nu l_{n}>\sqrt[3]{n}\right) \leqslant \mathbf{P}\left(S_{l_{n}}^{(\nu)}>(\nu+1) l_{n}\right) \leqslant \exp \left(-\psi^{(\nu)}\left(\nu+1 ; 2^{-1}\left(\nu+\mu^{+}\right)\right) l_{n}\right)$.

Аналогичная оценка верна для вероятностей $\mathbf{P}\left(S_{l_{n}}^{(\nu)}-\nu l_{n}<-\sqrt[3]{n}\right)$. Тем самым, интеграл $I_{2 n}$ убывает экспоненциально по переменной $l_{n}=[\sqrt[3]{n}]$, а соотношение (7.10) остается справедливым после замены в нем $I_{1 n}$ на $I_{n}$. Итак, получена правильная верхняя граница в (7.8).

d. Для вывода нижней границы в (7.8) воспользуемся разложением $V_{n}^{(\nu)}=V_{l_{n}}^{(\nu)}+e^{-\left(S_{l_{n}}^{(\nu)}-\nu l_{n}\right)-\nu l_{n}} \sum_{j=l_{n}+1}^{n} e^{-\left(S_{j}^{(\nu)}-S_{l_{n}}^{(\nu)}\right)}, \quad l_{n}=[\sqrt[3]{n}], \quad m_{n}=n-l_{n}$.

Разбивая блуждание $S_{j}^{(\nu)}$ на участки $j \leqslant l_{n}$ и $j \in\left[l_{n}+1, n\right]$, представим вероятность в левой части (7.8) в виде двойного интеграла по мере $\mu_{l_{n}}^{(\nu)}(d y, d z)$ от выражения

$\mathbf{P}\left(S_{m_{n}}^{(\nu)}-\nu\left(m_{n}+k\right)+y \in[x, x+\Delta], z+e^{-y-\nu l_{n}} V_{m_{n}}^{(\nu)} \leqslant v, L_{m_{n}}^{(\nu)}>-y-\nu l_{n}\right)$.

Выведем для него оценку снизу, ограничив интегрирование областью $\left\{|y| \leqslant \nu l_{n} / 2, z \leqslant v-\delta\right\}$, где $\delta>0$ будет выбираться сколь угодно малым. В указанной области имеем: $\left\{z+e^{-y-\nu l_{n}} V_{m_{n}}^{(\nu)} \leqslant v\right\} \supseteq\left\{V_{m_{n}}^{(\nu)} \leqslant \delta e^{\nu l_{n} / 2}\right\}$, $\left\{L_{m_{n}}^{(\nu)}>-y-\nu l_{n}\right\} \supseteq\left\{L_{m_{n}}^{(\nu)}>-\nu l_{n} / 2\right\}$, так что для вероятности (7.11) получаем следующую границу снизу:

$\mathbf{P}\left(S_{m_{n}}^{(\nu)}-\nu\left(m_{n}+k\right)+y \in[x, x+\Delta]\right)-\mathbf{P}\left(V_{m_{n}}^{(\nu)}>\delta e^{\nu l_{n} / 2}\right)-\mathbf{P}\left(L_{m_{n}}^{(\nu)} \leqslant-\nu l_{n} 2^{-1}\right)$.

Интеграл от первого слагаемого в (7.12) равен

$$
\int_{-\nu l_{n} / 2}^{\nu l_{n} / 2} \mathbf{P}\left(S_{m_{n}}^{(\nu)}-\nu\left(m_{n}+k\right)+y \in[x, x+\Delta]\right) \mu_{l_{n}}^{(\nu)}(d y ;(0, v-\delta])=: I_{1 n}^{\prime} .
$$

Он оценивается тем же способом, что и интеграл $I_{1 n}$. В результате приходим к соотношению (7.10), в котором $I_{1 n}$ заменяется на $I_{1 n}^{\prime}$, а событие $\left\{V_{\infty}^{(\nu)} \leqslant v\right\}-$ на событие $\left\{V_{\infty}^{(\nu)} \leqslant v-\delta\right\}$. Таким образом, для получения правильной нижней границы в (7.8) остается убедиться, что двумя другими слагаемыми в (7.12) можно пренебречь. Последнее слагаемое в (7.12) убывает экспоненциально по переменному $l_{n}$ в силу, например, неравенства из п. 2d. Аналогичное заключение для среднего члена в (7.12) вытекает из нижеследующей оценки.

е. Положим для краткости $\gamma:=\nu / 2$ и заметим, что

$$
\left\{\min _{0 \leqslant j \leqslant n}\left(S_{j}^{(\nu)}-\gamma j\right)>-y\right\} \subseteq\left\{V_{n}^{(\nu)} \leqslant \sum_{j=0}^{n} e^{-\gamma j+y} \leqslant\left(1-e^{-\gamma}\right) e^{y}\right\} .
$$


Следовательно, событие $\left\{V_{n}^{(\nu)}>\left(1-e^{-\gamma}\right) e^{y}\right\}$ влечет $\left\{\min _{0 \leqslant j \leqslant n}\left(S_{j}^{(\nu)}-\right.\right.$ $\gamma j) \leqslant-y\}$. Вероятность последнего события оценивается сверху по неравенству из п. $2 \mathbf{d}$ величиной $e^{p_{\gamma}^{(\nu)} y}$, где $p_{\gamma}^{(\nu)}:=\inf \left\{\lambda: \theta^{(\nu)}(\lambda) e^{-\lambda \gamma} \leqslant 1\right\}<0$. Таким образом, средняя вероятность в (7.12) убывает экспоненциально по переменному $l_{n}$, и соотношение (7.8) полностью доказано.

Выведем аналогичную оценку для $\mathbf{P}\left(V_{n}^{(\nu)}>v, L_{n}^{(\nu)}=0\right)$. Имеем:

$$
\left\{\min _{0 \leqslant j \leqslant n}\left(S_{j}^{(\nu)}-\gamma j\right)>-v, L_{n}^{(\nu)}=0\right\} \subseteq\left\{V_{n}^{(\nu)} \leqslant \frac{v}{\gamma}+1+\sum_{j>v / \gamma} e^{v-\gamma j}\right\},
$$

так что событие $\left\{V_{n}^{(\nu)}>v / \gamma+a_{\gamma}\right\}, a_{\gamma}:=1+e^{-\gamma}\left(1+e^{-\gamma}\right)^{-1}$, влечет событие $\left\{\min _{0 \leqslant j \leqslant n}\left(S_{j}^{(\nu)}-\gamma j\right) \leqslant-v\right\}$. Таким образом, для указанной вероятности получаем верхнюю границу $\exp \left(p_{\gamma}^{(\nu)} \gamma\left(v-a_{\gamma}\right)\right)$, экспоненциально убывающую по $v$.

f. Введем обозначение $J_{n, m}(A \times B ; u)$ для интеграла $J_{n, m}(u) \equiv$ $J_{n, m}((-\infty, \infty) \times(0, \infty) ; u)$, суженного на область $A \times B$ (см. $\left.(7.6)\right)$, и положим

$\widehat{J}(A \times B ; u):=\iint_{A \times B} \frac{1}{u+v} \exp \left(-\lambda^{*} x-\frac{e^{-x}}{u+v}\right) d x d \mathbf{P}\left(V_{\infty}^{\left(\mu^{*}\right)} \leqslant v, L_{\infty}^{\left(\mu^{*}\right)}=0\right)$.

Приступим к доказательству того, что при $n \rightarrow \infty$

$$
J_{n, m}(u) \sim\left(\sigma\left(\lambda^{*}\right) \sqrt{2 \pi n}\right)^{-1} \exp \left\{-\frac{1}{2}\left(\frac{\mu^{*} m}{\sigma\left(\lambda^{*}\right) \sqrt{n}}\right)^{2}\right\} \widehat{J}(u),
$$

$\widehat{J}(u):=\widehat{J}((-\infty, \infty) \times(0, \infty) ; u)$, равномерно по $m=O\left(n^{5 / 8}\right), u>1$. Соотношение (7.13) получается формальной заменой меры в интеграле (7.6) ее асимптотическим выражением, получаемым из (7.8). Поскольку эквивалентность (7.8) справедлива не во всей области изменения переменной $x$, то мы сузим область интегрирования в обоих интегралах $J_{n, m}$ и $\widehat{J}$, а затем докажем, что остатками можно пренебречь.

Заметим, что соотношение (7.13) выполняется, если заменить в нем $J_{n, m}(u)$ на $J_{n, m}([-a, a] \times(0, b] ; u)$ и аналогичную замену провести с $\widehat{J}(u)$, где $b>0$ - произвольная точка непрерывности функции распределения $\mathbf{P}\left(V_{\infty}^{\left(\mu^{*}\right)} \leqslant v, L_{\infty}^{\left(\mu^{*}\right)}=0\right), a>0-$ любое. Действительно, из (7.8) следует, что меры

$$
\sqrt{2 \pi n} \exp \left\{\frac{1}{2}\left(\frac{m}{\sigma\left(\lambda^{*}\right) \sqrt{n}}\right)^{2}\right\} \mathbf{P}\left(S_{n-m}^{\left(\mu^{*}\right)}-\mu^{*} n \in d x, V_{n-m}^{\left(\mu^{*}\right)} \in d v ; L_{n-m}^{\left(\mu^{*}\right)}=0\right)
$$

на $[-a, a] \times(0, b]$ слабо сходятся при $n \rightarrow \infty$ к мере $\sigma\left(\lambda^{*}\right)^{-1} d x \times \mathbf{P}\left(V_{\infty}^{\left(\mu^{*}\right)} \in\right.$ $\left.d v ; L_{\infty}^{\left(\mu^{*}\right)}=0\right)$ равномерно по $m \leqslant n^{5 / 8}$, а семейство подынтегральных функций в $(7.6)$ на $[-a, a] \times(0, b]$ равностепенно непрерывно по $u$. 
Далее заметим, что в области $[-a, a] \times(b, \infty)$ подынтегральная функция в (7.6) ограничена сверху величиной $b^{-1} e^{\lambda^{*} a}$. Вынесем эту оценку за знак интеграла, а то, что останется, равно

$$
\mathbf{P}\left(S_{n-m}^{\left(\mu^{*}\right)}-\mu^{*} n \in[-a, a], V_{n-m}^{\left(\mu^{*}\right)}>b, L_{n-m}^{\left(\mu^{*}\right)}=0\right) \leqslant \mathbf{P}\left(S_{n-m}^{\left(\mu^{*}\right)}-\mu^{*} n \in[-a, a]\right) .
$$

Применяя неравенство из п. 2g, получаем оценку:

$$
J_{n, m}([-a, a] \times(b, \infty) ; u) \leqslant b^{-1} e^{\lambda^{*} a}\left(2 a+\delta_{n-m}\right)\left(\sigma\left(\lambda^{*}\right) \sqrt{2 \pi(n-m)}\right)^{-1},
$$

меньшую $\varepsilon / \sqrt{n}$ для любых $a, \varepsilon>0$, достаточно большого $b, m \leqslant n^{5 / 8}$.

В области $(a, \infty) \times(0, \infty)$ подынтегральную функцию в $(7.6)$ ограничим сверху (при $u>1$ ) функцией $e^{-\lambda^{*} x}$ и отбросим событие $\left\{L_{n-m}^{\left(\mu^{*}\right)}=0\right\}$ :

$$
J_{n, m}((a, \infty) \times(0, \infty) ; u) \leqslant \int_{a}^{\infty} e^{-\lambda^{*} x} \mathbf{P}\left(S_{n-m}^{\left(\mu^{*}\right)}-\mu^{*} n \in d x\right) .
$$

Интеграл в правой части (7.14) оценим, переходя к интегральным суммам, как это делается в п. 4f, и используя неравенство (см. п. 2g)

$$
\mathbf{P}\left(S_{n-m}^{\left(\mu^{*}\right)}-\mu^{*} n \in[x, x+\Delta]\right) \leqslant\left(\Delta+\delta_{n-m}\right)\left(\sigma\left(\lambda^{*}\right) \sqrt{2 \pi(n-m)}\right)^{-1} .
$$

В результате получим для интеграла (7.14) при $m \leqslant n^{5 / 8}$ и достаточно большом $a$ границу сверху $\varepsilon / \sqrt{n}$.

Область $(-\infty,-a) \times(0, \infty)$ пересечем с множеством $\{(x, v): v \leqslant$ $\left.|x|^{-2} e^{-x}\right\}$ и обозначим полученное подмножество через $D$. Подынтегральную функцию в $J_{n, m}(D ; u)$ оценим сверху величиной $\exp \left(-\lambda^{*} x-x^{2}\right)$ и получим:

$$
J_{n, m}(D ; u) \leqslant \int_{-\infty}^{-a} \exp \left(-\lambda^{*} x-x^{2}\right) \mathbf{P}\left(S_{n-m}^{\left(\mu^{*}\right)}-\mu^{*} n \in d x\right) .
$$

Интеграл (7.15) оценивается сверху подобно (7.14) величиной $\varepsilon / \sqrt{n}$ для любого $\varepsilon>0$ и достаточно большого $a$. Остается оценить $J_{n, m}(\bar{D} ; u)$.

g. Выведем оценку сверху для вероятности

$$
\mathbf{P}\left(S_{n}^{(\nu)}-\nu n \in[x, x+\Delta], V_{n}^{(\nu)}>v, L_{n}^{(\nu)}=0\right) .
$$

Используя подход и обозначения п. е, имеем:

$$
\begin{aligned}
& \left\{\min _{0 \leqslant j \leqslant n}\left(S_{j}^{(\nu)}-\gamma j\right)>-y, L_{n}^{(\nu)}=0\right\} \subseteq\left\{V_{n}^{(\nu)} \leqslant \frac{y}{\gamma}+1+\sum_{j>y / \gamma} e^{y-\gamma j}\right\}, \\
& \mathbf{P}\left(S_{n}^{(\nu)}-\nu n \in[x, x+\Delta], V_{n}^{(\nu)}>y \gamma^{-1}+a_{\gamma}, L_{n}^{(\nu)}=0\right) \\
& \quad \leqslant \mathbf{P}\left(S_{n}^{(\nu)}-\nu n \in[x, x+\Delta], \min _{0 \leqslant j \leqslant n}\left(S_{j}^{(\nu)}-\gamma j\right) \leqslant-y\right), \quad y>0 .
\end{aligned}
$$


Введем случайный момент $\tau_{y} \leqslant \infty$ первого пересечения блужданием $S_{j}^{(\nu)}-\gamma j$ границы $-y$ и представим вероятность (7.17) в виде

$$
\sum_{k=1}^{n} \mathbf{E}\left\{\mathbb{I}_{\left\{\tau_{y}=k\right\}} \mathbf{P}\left(S_{n}^{(\nu)}-\nu n \in[x, x+\Delta] \mid S_{k}^{(\nu)}\right)\right\},
$$

где $\mathbb{I}_{\{\cdot\}}$ обозначает индикатор события, заключенного в скобки. Ограничим в (7.16), (7.18) значения $x$ неравенством $x>-(\nu-\alpha) n, \nu>\alpha>\gamma$. При $k \leqslant(1-\varepsilon) n, \varepsilon>0$, условную вероятность в (7.18) оценим сверху с помощью неравенства из п. 2g:

$$
\mathbf{P}\left(S_{n}^{(\nu)}-S_{k}^{(\nu)}-\nu n \in\left[-S_{k}^{(\nu)}+x,-S_{k}^{(\nu)}+x+\Delta\right] \mid S_{k}^{(\nu)}\right) \leqslant \frac{\Delta+\delta_{n-k}}{\sigma\left(\lambda_{\nu}\right) \sqrt{2 \pi(n-k)}} .
$$

При $\tau_{y}=k,(1-\varepsilon) n<k \leqslant n-1$, имеем: $S_{k}^{(\nu)} \leqslant \gamma k-y<\gamma k$, так что при $x>-(\nu-\alpha) n$ условная вероятность в (7.18) не превосходит

$$
\mathbf{P}\left(S_{n-k}^{(\nu)}-\nu n>-\gamma k-(\nu-\alpha) n\right) \leqslant \mathbf{P}\left(S_{n-k}^{(\nu)}>(\alpha-\gamma) n\right) .
$$

K правой вероятности в (7.20) применим первое из неравенств п. 2с и получим для нее следующую границу сверху:

$$
\exp \{-\lambda(\alpha-\gamma) n\} \theta^{(\nu)}(\lambda)^{n-k} \leqslant \exp \left\{-\left(\lambda(\alpha-\gamma)+\varepsilon \ln \theta^{(\nu)}(\lambda)\right) n\right\}
$$

для $\lambda \in\left(0, \lambda^{+}-\lambda_{\nu}\right)$. Фиксируя $\lambda$, возьмем $\varepsilon$ достаточно малым и получим, что граница (7.21) убывает экспоненциально быстро по $n$.

Заметим, что событие $\left\{\tau_{y}=n\right\}$ влечет $\left\{S_{n}^{(\nu)}<\gamma n\right\}$ и несовместно с событием $\left\{S_{n}^{(\nu)}-\nu n \in[x, x+\Delta]\right\}$ при $x \geqslant-(\nu-\alpha) n$. Таким образом, из оценок $(7.19),(7.21)$ вытекает, что вероятность (7.17) при $x \geqslant-(\nu-\alpha) n$ не превосходит

$$
c_{\nu} \Delta n^{-1 / 2} \mathbf{P}\left(\tau_{y} \leqslant n\right)=c_{\nu} \Delta n^{-1 / 2} \mathbf{P}\left(\min _{0 \leqslant j \leqslant n}\left(S_{j}^{(\nu)}-\gamma j\right) \leqslant-y\right)
$$

при некотором $c_{\nu}>0$. Вероятность в $(7.22)$ в силу неравенства из п. $2 \mathbf{d}$ (ср. п. е) не превосходит $\exp \left(p_{\gamma}^{(\nu)} y\right), p_{\gamma}^{(\nu)}<0$. В итоге вероятность $(7.16)$ оценивается сверху при $x \geqslant-(\nu-\alpha) n$ величиной $b_{\nu} \Delta n^{-1 / 2} \exp \left(-d_{\gamma}^{(\nu)} v\right)$, $d_{\gamma}^{(\nu)}>0$.

h. Подынтегральную функцию в $J_{n, m}(\bar{D} ; u)$ оценим сверху функцией $e^{-\lambda^{*} x}$, а область интегрирования по $x$, с учетом того, что $S_{n-m}^{\left(\mu^{*}\right)} \geqslant 0$ при условии события $\left\{L_{n-m}^{\left(\mu^{*}\right)}=0\right\}$, разобьем на две части: $\left[-\mu^{*} n,-\left(\mu^{*}-\alpha\right) n\right]$, $0<\alpha<\mu^{*}$, и $\left(-\left(\mu^{*}-\alpha\right) n,-a\right)$. Интеграл по первой части не превосходит

$$
\alpha n e^{\lambda^{*} \mu^{*} n} \mathbf{P}\left(V_{n-m}^{\left(\mu^{*}\right)}>\left(\left(\mu^{*}-\alpha\right) n\right)^{-2} e^{\left(\mu^{*}-\alpha\right) n}, L_{n-m}^{\left(\mu^{*}\right)}=0\right) .
$$

В п. е показано, что вероятность в (7.23) убывает как двойная экспонента по $n$, так что этим интегралом можно пренебречь. Интеграл по второй 
части заменим верхней суммой Дарбу-Стилтьеса по разбиению $x_{k}=$ $-a-k \Delta, v_{l}=a^{-2} e^{a}+l \Delta, k, l=0,1,2, \ldots$ В результате получим для него следующую оценку сверху при $a \geqslant 2$ :

$$
\sum_{k} e^{-\lambda^{*} x_{k}} \mathbf{P}\left(S_{n-m}^{\left(\mu^{*}\right)}-\mu^{*} n \in\left(x_{k}, x_{k-1}\right], V_{n-m}^{\left(\mu^{*}\right)}>\frac{1}{x_{k}^{2}} e^{-x_{k}}, L_{n-m}^{\left(\mu^{*}\right)}=0\right),
$$

суммирование в $(7.24)$ ведется по $k$ таким, что $x_{k} \geqslant-\left(\mu^{*}-\alpha\right) n-\Delta$. Так как при этом $\mu^{*} m+x_{k} \geqslant-\left(\mu^{*}-\alpha\right)(n-m)-\Delta$, то к вероятностям в $(7.24)$ применима оценка п. g, подставляя которую, получим:

$$
b_{\mu^{*}} e^{\lambda^{*} \Delta} \frac{1}{\sqrt{n}} \sum_{k} e^{-\lambda^{*} x_{k-1}} \exp \left(-d_{\gamma}^{\left(\mu^{*}\right)} \frac{1}{x_{k}^{2}} e^{-x_{k}}\right) \Delta .
$$

Сумма в (7.25) является нижней суммой Дарбу (при $a \geqslant 2$ ) для интеграла Римана от функции $\exp \left(-\lambda^{*} x-d_{\gamma}^{\left(\mu^{*}\right)}|x|^{-2} e^{-x}\right)$, который, очевидно, конечен и за счет выбора достаточно большого $a>0$ может быть сделан меньше любого $\varepsilon>0$. Таким образом, для интеграла $J_{n, m}(\bar{D} ; u)$ получена оценка сверху $\varepsilon / \sqrt{n}$.

Для доказательства соотношения (7.13) осталось убедиться, что $\widehat{J}(u)<\infty$. Проводя замену $y=e^{-x}(u+v)^{-1}$, получаем:

$$
\int_{-\infty}^{\infty} \exp \left(-\lambda^{*} x-e^{-x}(u+v)^{-1}\right) d x=(u+v)^{\lambda^{*}} \Gamma\left(\lambda^{*}\right),
$$

где $\Gamma(\lambda):=\int_{0}^{\infty} y^{\lambda-1} e^{-y} d y$,

$$
\widehat{J}(u)=\Gamma\left(\lambda^{*}\right) \int_{0}^{\infty}(u+v)^{\lambda^{*}-1} d \mathbf{P}\left(V_{\infty}^{\left(\mu^{*}\right)} \leqslant v, L_{\infty}^{\left(\mu^{*}\right)}=0\right)<\infty,
$$

поскольку хвосты $\mathbf{P}\left(V_{\infty}^{\left(\mu^{*}\right)}>v, L_{\infty}^{\left(\mu^{*}\right)}=0\right)$ убывают экспоненциально по $v$ (см. п. е). Отметим, что функция $\widehat{J}(u)$ непрерывна и ограничена, в чем легко убедиться, интегрируя (7.26) по частям.

i. Заменим $J_{n, m}(u)$ под интегралом $(7.7)$ при $m \leqslant n^{5 / 8}$ на равномерно по $m$ эквивалентное выражение (7.13) и запишем результат в виде

$$
\begin{gathered}
e^{-\psi\left(\mu^{*}\right) n} \theta\left(\lambda^{*}\right)^{-m}\left(\sigma\left(\lambda^{*}\right) \sqrt{2 \pi n}\right)^{-1} \exp \left\{-\left(\mu^{*} m\right)^{2}\left(2 \sigma\left(\lambda^{*}\right)^{2} n\right)^{-1}\right\} \\
\times \iint e^{y} \widehat{J}(u) \mathbf{P}\left(S_{m} \in d y, U_{m} \in d u ; S_{j}<0,1 \leqslant j \leqslant m\right) .
\end{gathered}
$$

Перепишем интеграл в (7.27) как $m$-кратный и сделаем в нем замену меры $\prod_{i=1}^{m} d F^{\left(\mu_{1}\right)}\left(x_{i}\right)=\theta(1)^{-m} e^{-s_{m}} \prod_{i=1}^{m} d F\left(x_{i}\right)$ :

$$
\begin{gathered}
\theta(1)^{m} \int_{\left\{s_{j}<0,1 \leqslant j \leqslant m\right\}} \ldots \int_{j=0} \widehat{J}\left(\sum_{j=0}^{m} e^{s_{j}}\right) \prod_{i=1}^{m} d F^{\left(\mu_{1}\right)}\left(x_{i}\right)=\theta(1)^{m} \int_{0}^{\infty} \widehat{J}(u) \mathbf{P}\left(U_{m}^{\left(\mu_{1}\right)} \in d u\right. \\
\left.S_{j}^{\left(\mu_{1}\right)}<0,1 \leqslant j \leqslant m\right), \quad U_{m}^{(\nu)}:=\sum_{j=0}^{m} e^{S_{j}^{(\nu)}}
\end{gathered}
$$


Интеграл в (7.28) сходится к $\mathbf{E}\left(\widehat{J}\left(U_{\infty}^{\left(\mu_{1}\right)}\right) ; S_{j}^{\left(\mu_{1}\right)}<0, j \geqslant 1\right)=: H$ при $m \rightarrow \infty$. В самом деле, так как $\mathbf{E} X^{\left(\mu_{1}\right)}=\mu_{1}<0$, то $U_{m}^{\left(\mu_{1}\right)} \rightarrow U_{\infty}^{\left(\mu_{1}\right)}$ п.н., $\max _{1 \leqslant j \leqslant m} S_{j}^{\left(\mu_{1}\right)} \rightarrow \max _{j \geqslant 1} S_{j}^{\left(\mu_{1}\right)}$ п.н., $m \rightarrow \infty$, причем обе последовательности неубывающие. Следовательно, функции распределения $\mathbf{P}\left(U_{m}^{\left(\mu_{1}\right)} \leqslant\right.$ $u, \max _{1 \leqslant j \leqslant m} S_{j}^{\left(\mu_{1}\right)} \leqslant z$ ) сходятся к предельной при всех $u, z$. Остается напомнить, что функция $\widehat{J}(u)$ непрерывна и ограничена.

Выберем $b_{n} \rightarrow \infty, b_{n}=o(\sqrt{n}), n \rightarrow \infty$. Члены суммы (7.4) эквивалентны

$$
H e^{-\psi\left(\mu^{*}\right) n}\left(\sigma\left(\lambda^{*}\right) \sqrt{2 \pi n}\right)^{-1} \exp \left\{-\left(\mu^{*} m\right)^{2}\left(2 \sigma\left(\lambda^{*}\right)^{2} n\right)^{-1}\right\}
$$

при $m \geqslant b_{n}$, а при $m<b_{n}$ выражения (7.29), домноженные на подходящую константу, дают верхнюю границу для соответствующих членов (7.4). Суммируя (7.29) по $m<b_{n}$, получаем $O\left(b_{n} / \sqrt{n}\right) e^{-\psi\left(\mu^{*}\right) n}=$ $o(1) e^{-\psi\left(\mu^{*}\right) n}$. Сумма выражений (7.29) по $m \in\left[b_{n}, n^{5 / 8}\right]$ эквивалентна $\left(2 \mu^{*}\right)^{-1} H e^{-\psi\left(\mu^{*}\right) n}$. Доказательство теоремы 4 завершено. Заметим, что ввиду формулы (7.26)

$$
H=\Gamma\left(\lambda^{*}\right) \mathbf{E}\left\{\left(\widetilde{U}_{\infty}^{\left(\mu_{1}\right)}+V_{\infty}^{\left(\mu^{*}\right)}\right)^{\lambda^{*}-1} ; \widetilde{S}_{j}^{\left(\mu_{1}\right)}<0, j \geqslant 1, L_{\infty}^{\left(\mu^{*}\right)}=0\right\},
$$

где случайные блуждания $S_{j}^{\left(\mu_{1}\right)}$ и $S_{j}^{\left(\mu^{*}\right)}$ считаются независимыми.

j. Доказательство теоремы 5 почти дословно следует доказательству теоремы 4. Оценки пп. $\mathbf{a}, \mathbf{c}, \mathbf{d}, \mathbf{e}$ вообще не связаны со значением $t=\mu^{*}$ и годятся для $t \in\left(0, \mu^{*}\right]$, причем они равномерны по $t \in\left[\varepsilon, \mu^{*}\right], \varepsilon>0$. Построения пп. $\mathbf{b}, \mathbf{f}, \mathbf{h}$ сохраняются с заменой $\mu^{*}$ на $t \in\left(0, \mu^{*}\right)$ и заменой области суммирования в (7.4) на $\left|m-n \hat{s}_{t}\right| \leqslant n^{5 / 8}$. Для $t \in\left[\varepsilon, \mu^{*}\left(1-n^{-3 / 8}\right)\right]$ это приводит к удвоению постоянного множителя в асимптотике (3.3) по сравнению с (3.2). Для $t_{n} \in\left[\mu^{*}\left(1-n^{-3 / 8}\right), \mu^{*}\right]$ соответствующая асимптотика дается правой частью соотношения (3.3) при $t=t_{n}$ с дополнительным множителем $\Phi\left(\sqrt{n} \widehat{s}_{t_{n}} / b_{\mu^{*}}\right)$ (см. (4.33)).

8. Заключительные замечания. Техника настоящей работы позволяет уточнить асимптотическую картину поведения ВПСС при условии больших уклонений $\ln Z_{n}>t n$ в форме условных функциональных предельных теорем (см. [8] и цитированную там литературу). Различие в асимптотиках вероятностей больших уклонений при $t \leqslant \mu^{*}$ и $t>\mu^{*}$ проявляется при рассмотрении типичных траекторий, приводящих в каждом из этих случаев к большому уклонению. При $t>\mu^{*}$ большое уклонение обеспечивается за счет реализации среды, которая приводит к большому уклонению $S_{n}=t n+O_{p}(1)$. При условии $\ln Z_{n}>t n, t<\mu^{*}$, среда на участке $\left[0, T_{n}\right]$, где случайный момент $T_{n}$ асимптотически нормален со средним $\widehat{s}_{t} n$ и дисперсией $\sigma\left(\lambda_{t}\right)^{2} n$, благоприятствует невырождению ВПСС, а на участке $\left[T_{n}, n\right]$ она способствует большому уклонению по тому же типу, что и в случае $t>\mu^{*}$. 
Распространение результатов на случай произвольной дробнолинейной производящей функции $g(z)$ не представляет принципиальных затруднений. Теорема 1 о грубой асимптотике вероятностей больших уклонений переносится на случай производящей функции $g(z)$ общего вида. Точная асимптотика требует более детального анализа.

Пользуюсь возможностью выразить благодарность В. А. Ватутину за ценные обсуждения. Я также благодарен Г. Керстингу и Й. Гейгеру за внимание к работе и приглашение в свое время на рабочее совещание во Франкфурте, которое побудило меня заняться исследованием вероятностей больших уклонений для ВПСС.

Я чрезвычайно благодарен анонимному рецензенту за внимательное прочтение рукописи, выявление многих неточностей, как формального, так и содержательного характера, и советы по внесению в текст статьи соответствующих изменений. Большим уклонениям случайных блужданий и близких к ним марковских цепей уделялось в последнее десятилетие большое внимание в статьях и монографиях (в том числе и в соавторстве) А. А. Боровковым, К. А. Боровковым, А. А. Могульским, Д.А.Коршуновым, в которых были получены глубокие результаты. Рассматриваемый нами ВПСС попадает в класс асимптотически однородных марковских цепей в терминологии А. А. Боровкова. Однако, как это нередко бывает, специфика конкретной модели позволяет придти к более точным выводам.

\section{СПИСОК ЛИТЕРАТУРЫ}

1. Agresti A. On the extinction times of varying and random environment branching processes. - J. Appl. Probab., 1975, v. 12, № 1, p. 39-46.

2. Петров В.В. О вероятностях больших уклонений сумм независимых случайных величин. - Теория вероятн. и ее примен., 1965 , т. 10, в. 2, с. 310-322.

3. Козлов М. В. О больших уклонениях ветвящихся процессов в случайной среде: геометрическое распределение числа потомков. - Дискретн. матем., 2006, т. 18, № 2 , c. $29-47$.

4. Kesten $H$. Random difference equations and renewal theory for products of random matrices. - Acta Math., 1973, v. 131, p. 207-248.

5. Боровков A. А. Вероятностные процессы в теории массового обслуживания. М: Наука, 1972, 368 с.

6. Феллер В. Введение в теорию вероятностей и ее приложения, т. 2. М: Мир, 1984.

7. Ибрагимов И.А., Линник Ю.В. Независимые и стационарно связанные величины. М: Наука, 1965, 524 с.

8. Afanasyev V.I., Geiger J., Kersting G., Vatutin V.A. Criticality for branching processes in random environment. - Ann. Probab., 2005, v. 33, № 2, c. 645-673.

Поступила в редакцию

5.VI.2008

Исправленный вариант

4.XII.2008 